\title{
Dopamine Receptor Blockade Modulates the Rewarding and Aversive Properties of Nicotine via Dissociable Neuronal Activity Patterns in the Nucleus Accumbens
}

\author{
Ninglei Sun', Steven R Laviolette*, 1,2,3 and Addiction Research Group \\ 'Department of Anatomy and Cell Biology, The Schulich School of Medicine and Dentistry, University of Western Ontario, London, ON, Canada; \\ ${ }^{2}$ Department of Psychiatry, The Schulich School of Medicine and Dentistry, University of Western Ontario, London, ON, Canada; ${ }^{3}$ Department of \\ Psychology, The Schulich School of Medicine and Dentistry, University of Western Ontario, London, ON, Canada
}

\begin{abstract}
The mesolimbic pathway comprising the ventral tegmental area (VTA) and projection terminals in the nucleus accumbens (NAc) has been identified as a critical neural system involved in processing both the rewarding and aversive behavioral effects of nicotine. Transmission through dopamine (DA) receptors functionally modulates these effects directly within the NAc. Nevertheless, the neuronal mechanisms within the NAc responsible for these bivalent behavioral effects are presently not known. Using an unbiased conditioned place preference procedure combined with in vivo neuronal recordings, we examined the effects of nicotine reward and aversion conditioning on intra-NAc neuronal sub-population activity patterns. We report that intra-VTA doses of nicotine that differentially produce rewarding or aversive behavioral effects produce opposite effects on sub-populations of fast-spiking interneurons (FSls) or medium spiny neurons (MSNs) within the shell region of the NAc (NAshell). Thus, while the rewarding effects of intra-VTA nicotine were associated with inhibition of FSI and activation of MSNs, the aversive effects of nicotine produced the opposite pattern of NAshell neuronal population activity. Blockade of DA transmission with a broad-spectrum DA receptor antagonist, $\alpha$-flupenthixol, strongly inhibited the spontaneous activity of NAshell FSIs, and reversed the conditioning properties of intra-VTA nicotine, switching nicotineconditioned responses from aversive to rewarding. Remarkably, DA receptor blockade switched intra-NAshell neuronal population activity from an aversion to a reward pattern, concomitant with the observed switch in behavioral conditioning effects.
\end{abstract}

Neuropsychopharmacology (2014) 39, 2799-28I5; doi:I0.1038/npp.20I4.130; published online 2 July 2014

\section{INTRODUCTION}

Nicotine possesses both rewarding and aversive stimulus properties, and can act directly within the ventral tegmental area (VTA) to produce bivalent, dose-dependent, rewarding, or aversive behavioral effects (David et al, 2006; Jorenby et al, 1990; Laviolette et al, 2008; Laviolette and van der Kooy, 2003). Considerable evidence implicates the mesolimbic dopamine (DA) system, comprising the VTA and its projections to the nucleus accumbens (NAc), as important regulators of nicotine's motivational effects (Corrigall et al 1992; 1994; Mansvelder et al, 2002; Nisell et al, 1994; Sellings et al, 2008; Tan et al, 2009; ). We have reported previously that whereas relatively lower concentrations of nicotine produced behaviorally aversive effects in the VTA, higher concentrations produced strong

*Correspondence: Dr SR Laviolette, Department of Anatomy and Cell Biology, The Schulich School of Medicine and Dentistry, University of Western Ontario, 468 Medical Science Building, London, ON, Canada N6A 5Cl, Tel: + 5196612111 ext. 80302, Fax: + 5196613936 E-mail: steven.laviolette@schulich.uwo.ca

Received I4 February 2014; revised 29 May 20I4; accepted 30 May 20I4; accepted article preview online 4 June 2014 rewarding effects, measured in place conditioning procedures (Laviolette and van der Kooy, 2003; 2004). Although the acute aversive effects of nicotine are DA dependent, following chronic nicotine exposure and dependence, the rewarding effects of nicotine are mediated through a DAdependent pathway (Corrigall et al, 1992; Tan et al, 2009). Thus, blockade of DA transmission blocks the acute aversive behavioral effects of systemic or intra-VTA nicotine as measured in either place conditioning procedures or other aversion assays, such as conditioned taste aversion (Laviolette and van der Kooy, 2003). Furthermore, blocking intra-NAc DA transmission or lesion of DA terminals within specific NAc sub-regions increases the reward salience of nicotine and switches the valence of both systemic or intra-VTA nicotine from aversive to rewarding (Laviolette and van der Kooy, 2003; Sellings et al, 2008). The importance of DA neurotransmission in nicotine's behavioral effects is further underscored by evidence, demonstrating that DA release is strongly increased by acute nicotine administration, by directly exciting VTA DAergic neurons and/or increasing release of DA in the terminal fields of the mesolimbic DAergic projections (Mansvelder et al, 2002; Nisell et al, 1994; Yin and French, 2000). 
However, the effects of DAergic transmission modulation on nicotine reward signaling and how specific neuronal sub-populations within the NAc may be involved in processing the bivalent behavioral properties of nicotine are not currently understood. Neurons within the NAc comprises a majority ( $95 \%)$ of medium spiny neurons (MSNs) and heterogeneous populations of interneurons, including fast-spiking interneurons (FSIs), GABAergic interneurons, and cholinergic interneurons, which provide functional feedforward inhibition to MSN populations (English et al, 2012; Gage et al, 2010; Koos and Tepper, 1999). These neuronal groups are important for rewarding and aversive motivational processing. Thus, reward-related behavioral conditioning is associated with inhibition of rodent FSIs, concomitant with increased MSN activity in vivo (Lansink et al, 2010). DA transmission exerts complex effects on NAc neuronal network dynamics wherein DA can strongly activate FSI populations (Bracci et al, 2002), in turn leading to GABAergic-mediated inhibition of MSN output pathways (Nisenbaum and Berger, 1992). We hypothesized that the DA-dependent, acute aversive behavioral effects of nicotine would modulate NAc neuronal network dynamics by preferentially activating FSI neuronal populations and concomitantly inhibiting MSN neuronal activity. In the present study, we report that the rewarding or aversive behavioral effects of nicotine are associated with opposing neuronal activation patterns in the shell of the NAc (NAshell). Remarkably, switching the aversive behavioral properties of nicotine into reward signals via DA receptor blockade completely reversed these neuronal activity patterns, demonstrating a novel neuronal network mechanism underlying the bivalent conditioning effects of nicotine.

\section{MATERIALS AND METHODS}

\section{Nicotine Conditioned Place Preference Procedure}

In Figure 1a, behavioral experimental procedures for nicotine conditioned place preference (CPP) training are schematically presented. An unbiased, fully counterbalanced CPP procedure was used, as described previously (Laviolette and van der Kooy, 2003). In brief, drug or saline vehicle was paired with one of two environments that differed in terms of color, texture, and smell. Following recovery from surgery, rats were randomly assigned to an experimental group. Conditioning boxes were $30 \times 30 \times 15$ inches (length by height) in dimension. One conditioning environment was white with a wire-mesh floor covered in woodchips. The alternate environment was black with a smooth Plexiglas floor wiped down with $2 \%$ acetic acid immediately a $\alpha$-FLU or VEHICLE PRE-TREATMENT $(+)$ INTRA-VTA NICOTINE/VEHICLE

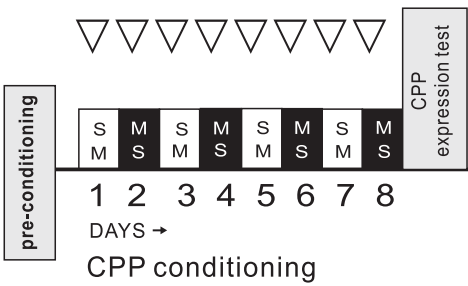

b

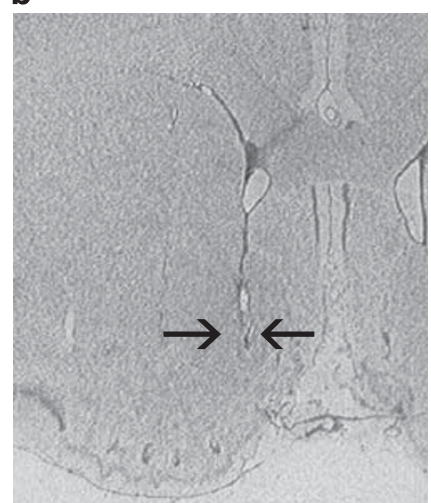

c

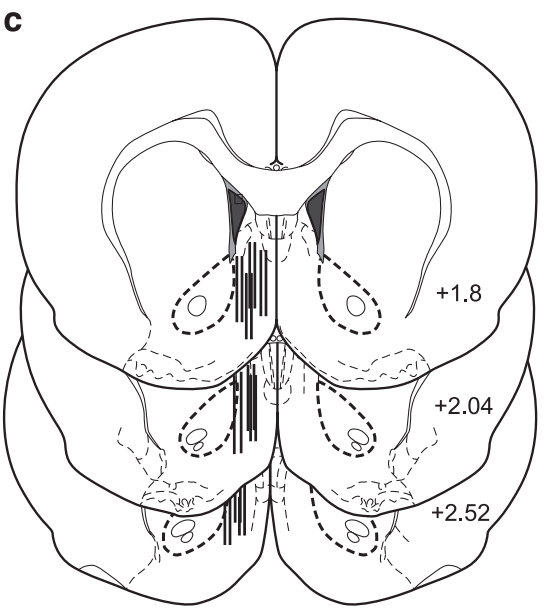

d

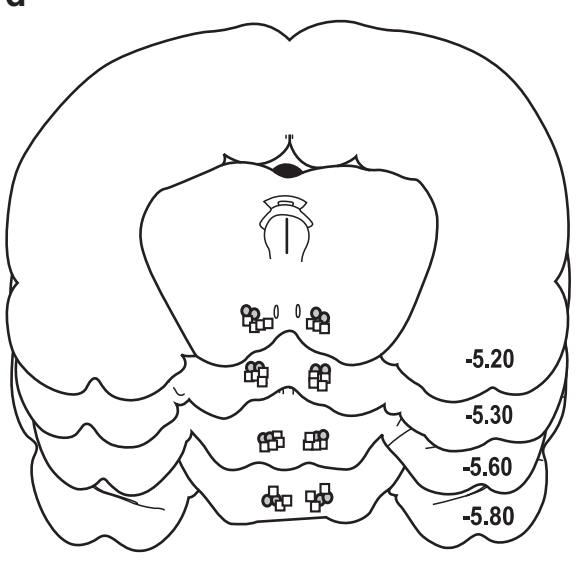

Figure I Experimental protocol and histological analysis. (a) Schematic summary of nicotine conditioned place preference behavioral procedure. (b) Microphotograph showing representative microwire placement in the shell region of the nucleus accumbens. (c) Schematic representation of typical unilateral intra-NAshell microwire placements. (d) Schematic summary of intra-ventral tegmental area (VTA) microinfusion locations. Gray circles indicate experimental group receiving intra-VTA nicotine at $24 \mathrm{nmol} / 0.5 \mu \mathrm{l}(n=8)$. White squares represent rats receiving intra-VTA nicotine $(0.008 \mathrm{nmol} / 0.5 \mu \mathrm{l})$ before $\alpha$-flupenthixol $(n=8)$ pre-treatment (see Materials and Methods section). NAshell, shell region of the NAc. 
before the animal was placed into it. Experimental treatments were fully counterbalanced within groups, and rats were randomly assigned to either the white or black colored boxes for drug/vehicle conditioning sessions. As has been reported previously, rats display no baseline preference for either of these environments (Laviolette and van der Kooy, 2003). In brief, during the CPP acquisition phase, rats received a total of four nicotine-environment and four vehicle-environment pairings over 8 days in a counterbalanced order. Two days following the acquisition phase, rats received a CPP recall test wherein the subject is placed on a narrow and neutral gray zone, and times spent in either of the previously conditioned environments are digitally recorded for $10 \mathrm{~min}$. All rats were tested in a drug-free state.

\section{Animals and Surgery}

All experimental procedures were performed in accordance with both institutional and Canadian Governmental animal care guidelines. All subjects used in these experiments were adult (350-400 gm) male Sprague-Dawley rats. Rats were individually housed in clear Plexiglas cages in a temperature-controlled room $\left( \pm 21^{\circ} \mathrm{C}\right)$ with access to food and water ad libitum throughout the duration of the experiments. Rats were anesthetized with a mixture of ketamine $(80 \mathrm{mg} / \mathrm{ml})$ and xylazine $(6 \mathrm{mg} / \mathrm{ml})$, and placed into a stereotaxic device (volume per body weight). Incisions were made in the scalp to expose the skull, and burr holes were drilled and the dura was removed overlying the NAc and VTA regions. Eight-channel microwire arrays (TuckerDavis) were slowly lowered unilaterally into the NAshell region using the following stereotaxic coordinates (Paxinos and Watson, 2005): (in $\mathrm{mm}$ ) from bregma: anteroposterior $(\mathrm{AP})=+2.2$ and lateral $(\mathrm{L})= \pm 1.2$; and $\operatorname{ventral}(\mathrm{V})=-7$ from the dural surface. For bilateral intra-VTA guide cannulae implantation, stainless steel guide cannulae (22 gauge; Plastics One) were implanted using the following stereotaxic coordinates (in $\mathrm{mm}$ ) at $10^{\circ}$. For the VTA: from bregma, $\mathrm{AP}=-5.0$ and $\mathrm{L}= \pm 2.3$; and from the dural surface, $\mathrm{V}=-8.0$. Jeweler's screws were attached to the skull surface, following which dental acrylic was applied to secure the microarray. Animals were allowed 10 days to recover from surgical procedures before any experiments were performed.

\section{Experimental Groups}

Four groups of rats were used in the present experiments. To determine the characteristics of a normally rewarding dose of intra-VTA nicotine $(24 \mathrm{nmol} / 0.5 \mu \mathrm{l})$ on intraNAshell neuronal activity patterns, the first experimental group received a previously established rewarding dose of intra-VTA nicotine $(24 \mathrm{nmol} / 0.5 \mu \mathrm{l})$ as described above $(n=8)$ following systemic vehicle pretreatment. A subsequent group $(n=7)$ received conditioning with this same rewarding dose of intra-VTA nicotine after pre-treatment with systemic $\alpha$-flupenthixol ( $\alpha$-flu), using a previously established dose of $0.8 \mathrm{mg} / \mathrm{kg}$; i.p.; previously shown to reverse the effects of both systemic or intra-VTA nicotine behaviorally aversive effects, as measured in the CPP procedure (Laviolette et al, 2008; Laviolette and van der Kooy,
2003, 2004; Tan et al, 2009). Having established rewardrelated NAshell neuronal activity parameters, we next examined the NAshell neuronal response patterns associated with a previously established, acutely aversive dose of intra-VTA nicotine $(0.008 \mathrm{nmol} / 0.5 \mu \mathrm{l} ; n=8)$ following systemic vehicle pre-treatment, and then again examined the effects of DA receptor blockade on intra-VTA nicotine aversion conditioning with an additional experimental group $(n=8)$.

\section{Multi-Unit Electrophysiological Recordings and Analysis}

In vivo microwire recording procedures were similar to those previously described (Sun et al, 2011; Sun and Laviolette, 2012). In brief, eight-channel microwire arrays (model MW8, Tucker-Davis) were used to record neuronal activity within the NAshell (Figure $1 \mathrm{~b}$ and c). Microwire arrays consisted of eight wires arranged in two rows, with each row separated by $500 \mu$ and the anterior-posterior wire placements separated by $50 \mu$ each. Thus, the dimensions of the eight-wire arrays were $200 \mu$ in length by $500 \mu$ in width. Microwire probes were connected to an RA-16PA preamplifier and then sent to a Pentusa Base Station (model RX5, Tucker-Davis). Neuronal spike signals were sampled at $25 \mathrm{kHz} / \mathrm{channel}$ with filter settings of $100 \mathrm{~Hz}$ (high-pass) and $5 \mathrm{kHz}$ (low-pass), and were then sent to a window discriminator/amplifier and an audio monitor. Neuronal activity data were simultaneously collected and monitored online using spike sorting software (Open Ex, TuckerDavis), and stored on computer for offline analysis. Spike waveforms were sorted and analyzed using built-in K-means analysis software (OpenSort, TDT) offline. Subsequent electrophysiological analysis was performed with NeuroExplorer (NEX Technology). Two sets of baseline neuronal activity were obtained from each rat. First, to ensure that there were no pre-existing differences in neuronal response rates during exposure to a particular conditioning environment, $10 \mathrm{~min}$ of recordings were performed in each of the conditioning environments, in a counterbalanced order, over 2 days, pre-conditioning. Next, baseline neuronal activity recordings were performed before the beginning of each behavioral training session within the home cage for $10 \mathrm{~min}$, following intra-VTA (Figure 1d) infusions of vehicle. These home-cage baselines were averaged within each group and used for subsequent comparison with activity rates during the conditioning sessions. We then compared baseline NAshell neuronal activity states across experimental recording sessions within each experimental phase (acquisition and recall). To distinguish NAc FSIs from presumptive MSNs, isolated units per channel were separated according to spike waveform, and firing frequency parameters were recorded during baseline (Figure 2). During offline analyses, isolated neuronal units (Figure 2a) were manually sorted based on these waveformshaped criteria, and interspike interval histograms (Figure 2b-e) were constructed for each isolated unit with NeuroExplorer. Sub-populations of neuronal units met previously established criteria for presumptive FSIs (Berke et al, 2004) or MSNs (Morra et al, 2010). MSN waveforms demonstrated longer duration (valley widths $>300 \mu \mathrm{s}$ ) and lower spontaneous baseline firing rates $(<5 \mathrm{~Hz})$. In 

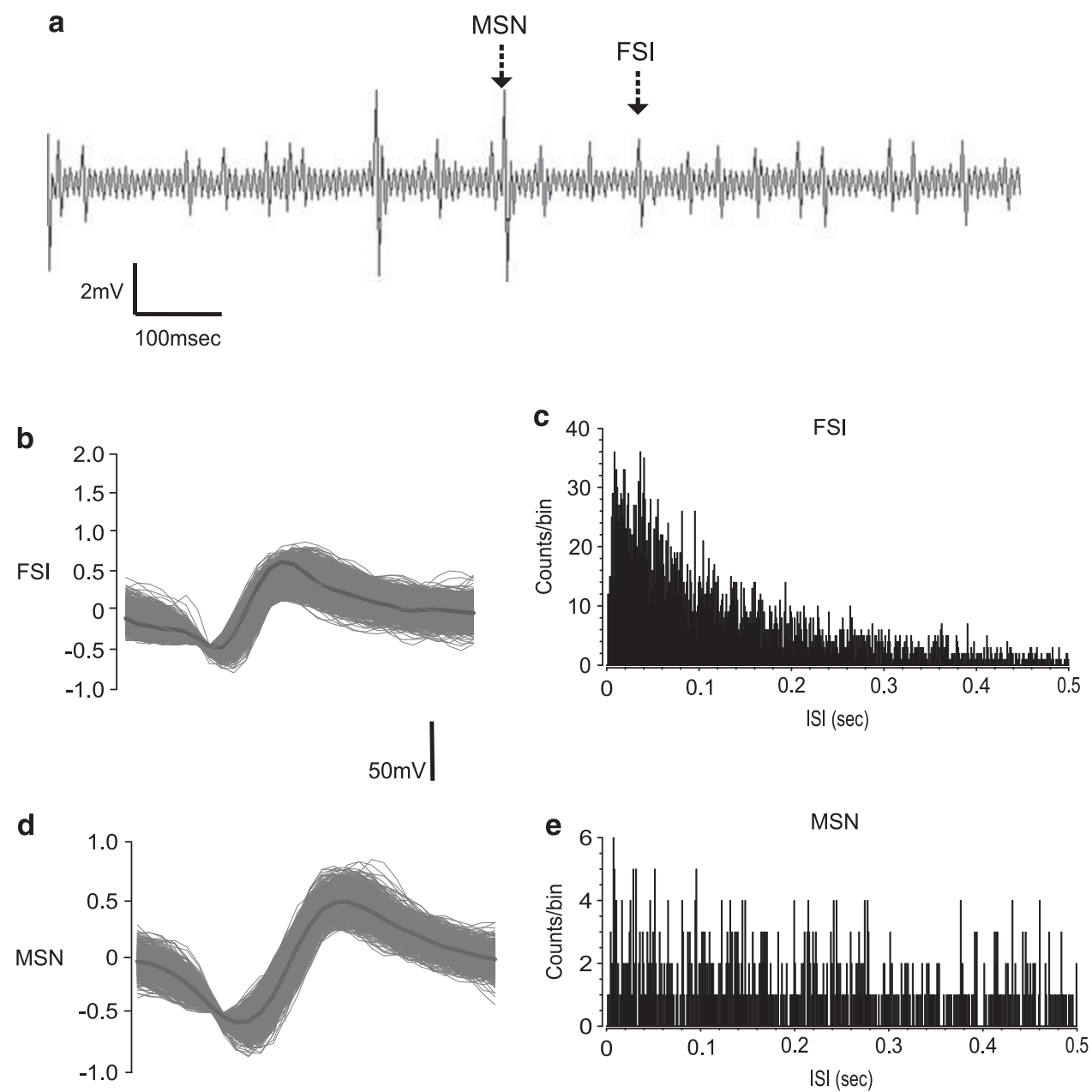

Figure 2 Analysis of in vivo intra-NAshell neuronal recordings. (a) Sample recording trace from an intra-NAshell microwire channel showing representative medium spiny neuron (MSN) and fast-spiking interneuron (FSI) neuronal traces. (b) Representative recording waveforms and interspike interval histogram for FSI ( $b$ and $c$ ) or MSNs ( $d$ and e) recorded during behavioral conditioning. NAshell, shell region of the NAc.

contrast, FSI waveforms demonstrated shorter durations (peak width $<265 \mu \mathrm{s}$ ) and higher spontaneous firing rates $(>5 \mathrm{~Hz})$.

\section{Drugs and Injection Procedures}

For intra-VTA nicotine experiments (Figure 1d), we selected two previously established concentrations of nicotine $(0.008 \mathrm{nmol} / 0.5 \mu \mathrm{l}$ or $24 \mathrm{nmol} / 0.5 \mu \mathrm{l})$ demonstrated to produce either robust conditioned place aversions (CPAs) or CPPs (Laviolette et al, 2008; Laviolette and van der Kooy, 2003, 2004; Tan et al, 2009), respectively. Nicotine tartrate (Sigma) was dissolved in physiological saline ( $\mathrm{pH}$ adjusted to 7.4), which served as the vehicle solution for all drugs used in these studies. Bilateral intra-VTA microinjections of nicotine or vehicle $(0.5 \mu \mathrm{l}$ volume per infusion) were performed over $1 \mathrm{~min}$ via plastic tubing (Millipore) connected to a 1- $\mu$ l Hamilton micro-syringe. Injectors were then left in place for an additional $1 \mathrm{~min}$ to ensure adequate diffusion from the injector tip. Infusions were performed immediately before placing rats into the assigned conditioning environments, as described previously (Laviolette et al, 2008; Tan et al, 2009). For experiments examining the effects of DA receptor blockade on intra-VTA nicotine conditioning, a broad-spectrum DA receptor antagonist, $\alpha$-flu (Tocris) was dissolved in physiological vehicle, and administered $2.5 \mathrm{~h}$ before intra-VTA nicotine conditioning sessions, as described previously (Laviolette and van der Kooy, 2003; Tan et al, 2009). This $\alpha$-flu pretreatment regimen produces no behavioral motivational effects in and of itself (Laviolette and van der Kooy, 2003), and is sufficient to block both pre- and post-synaptic D1/D2 receptors (Creese et al, 1976).

\section{Histological Analysis}

At the end of behavioral experiments, rats were anesthetized and intracardially perfused with isotonic vehicle, followed by $10 \%$ formalin. Brains were removed and post-fixed in a $25 \%$ sucrose solution. The brains were cut in $40 \mu \mathrm{m}$ sections and stained with Cresyl Violet. Microarray placements within the MPFC were then verified by light microscopy.

\section{Data Analysis}

All data are expressed as mean \pm SEM and were analyzed with one or two-way analysis of variance (ANOVA) or Student's $t$-tests where appropriate. Post hoc analyses were 
performed with Newman-Keuls or Fisher's LSD tests where appropriate. For post-experimental neuronal activity analyses during specific nicotine reward learning phases, neuronal sub-populations were further sub-classified according to recording channels yielding consistent waveforms across all trials within either the acquisition or extinction training sessions. K-means analysis of recorded waveforms across channels revealed consistent single-unit activity over experimental sessions (see Results section); however, given the long-term behavioral recordings inherent in these studies, each phase of the experimental procedure (acquisition, expression, and extinction) and individual recording sessions within each experimental phase were analyzed separately, as it is not possible to determine with certainty that a specific channel is picking up the same unit across days or experimental phase. Accordingly, we report ' $n$ ' sizes of sampled neuronal populations separately for each experimental phase reported in the results section.

\section{RESULTS}

\section{Histological Analyses}

Analyses of intra-NAshell microwire placements and bilateral intra-VTA cannulae placements revealed recording and infusions sites to be within the anatomical boundaries of the NAshell or the VTA, as defined by Paxinos and Watson (2005). In Figure 1b, we present a schematic representation of typical intra-NAshell microwire track locations. In Figure 1c, we present a microphotograph showing a typical intra-NAshell microwire track location. In Figure 1d, we present a schematic representation of representative, bilateral intra-VTA cannulae tip locations. For clarity, we present two experimental groups (gray circles indicate the group receiving intra-VTA nicotine at a higher dose of $24 \mathrm{nmol} / 0.5 \mu \mathrm{l}(n=8)$, whereas white squares represent rats receiving a lower dose of intra-VTA nicotine $(0.008 \mathrm{nmol} / 0.5 \mu \mathrm{l})$ before $\alpha$-flu $(n=8)$ pre-treatment (see Materials and Methods section).

\section{Neuronal Response Patterns in the NAshell During intra-VTA Nicotine Reward Learning Acquisition}

To determine how intra-NAshell neuronal activity may be modulated by an acutely rewarding dose of intra-VTA nicotine, we first examined how NAshell neuronal populations responded to nicotine reward learning using a previously established (Laviolette and van der Kooy, 2003; 2004; Tan et al, 2009) robustly rewarding dose of intra-VTA nicotine $(24 \mathrm{nmol} / 0.5 \mu \mathrm{l})$. For $\mathrm{MSN}(n=18)$ recordings, one-way ANOVA comparing neuronal frequencies during vehicle $v s$ nicotine conditioning sessions revealed a significant effect of conditioning session (vehicle $v s$ nicotine) on firing rates $(\mathrm{F}(8,134)=2.37 ; p<0.05)$. Post hoc analysis revealed that MSN firing rates were significantly increased relative to vehicle levels during the first and second conditioning trials ( $p$-values $<0.01$ and 0.05 , respectively; Figure 3a). Furthermore, MSN activity levels during nicotine sessions were significantly increased relative to baseline levels during the first, second, and fourth sessions ( $p$-values $<0.05$ ). In contrast, following
intra-VTA vehicle, MSN activity levels were increased significantly above baseline only during the fourth vehicle session $(p<0.05$; Figure 3a). Comparing MSN neuronal activity patterns across all conditioning sessions recorded in each separate conditioning environment revealed no differences in MSN activity across these environments independently of drug treatment $\left(t_{(17)}=1.00, p>0.05\right.$; Figure 3a, far right side). Furthermore, comparing MSN firing activity during pre-conditioning exposures to each conditioning environment alone (see Materials and Methods section) revealed no significant differences between environments $\left(t_{(17)}=0.78 ; p>0.05\right.$; data not shown).

For FSI recordings $(n=15)$, one-way ANOVA comparing neuronal frequencies during vehicle $v s$ nicotine conditioning sessions revealed a significant effect of conditioning session (vehicle $v s$ nicotine) on firing rates $(\mathrm{F}(8,314)=8.02$; $p<0.0001)$. Post hoc analysis revealed that FSI firing rates were significantly decreased relative to vehicle levels during the first and second conditioning trials ( $p$-values $<0.05$; Figure 3b) Further, FSI activity levels during nicotine sessions were significantly decreased relative to baseline levels during the first and second $(p$-values $<0.05)$ and third and fourth sessions ( $p$-values $<0.01$ ). In contrast, activity levels were significantly decreased below baseline during third and fourth vehicle sessions ( $p$-values $<0.01$; Figure $3 \mathrm{~b}$ ). Comparing FSI neuronal activity patterns across all conditioning sessions recorded in each separate conditioning environment revealed no differences in MSN activity across these environments independently of drug treatment $\left(t_{(14)}=0.94 ; p>0.05\right.$; Figure $3 \mathrm{~b}$, far right side). Furthermore, comparing FSI firing activity during preconditioning exposures to each conditioning environment alone (see Materials and Methods section) revealed no significant differences between environments $\left(t_{(14)}=0.68\right.$; $p>0.05$; data not shown).

Consistent with previous reports (Laviolette and van der Kooy, 2003; Tan et al, 2009), this dose of intra-VTA nicotine $(24 \mathrm{nmol} / 0.5 \mu \mathrm{l})$ produced a robust behavioral CPP $\left(t_{(7)}=2.24\right.$; $p<0.05$; Figure $3 c$ ), with rats spending significantly more time in the nicotine-paired environment during CPP testing. Post-test analysis comparing times spent in each of the separate conditioning environments (independently of treatment) revealed no significant bias toward either environment $\left(t_{(7)}=0.23 ; p>0.05\right.$; Figure $3 c$, inset). One-way ANOVA comparing MSN and FSI activity patterns during exposure to either vehicle or nicotine-paired environments during the initial CPP test (rats tested in a drug-free state, see Materials and Methods section) revealed a significant effect of group on frequency $(\mathrm{F}(2,47)=14.1 ; p<0.0001)$. Post hoc analysis revealed that whereas MSN activity during nicotine environment exposure was significantly increased relative to vehicle $(p<0.01)$, FSI activity was significantly suppressed $(p<0.05$; Figure $3 \mathrm{~d})$, revealing a similar MSN/ FSI activity pattern divergence during nicotine CPP reward memory recall to the patterns observed during acquisition training (Figure $3 a$ and $b$ ). In Figure $3 e$, we present sample MSN and FSI overlaid recording traces showing a 10-min baseline activity epoch followed by a 10 -min post-infusion recording following an intra-VTA nicotine $(24 \mathrm{nmol} / 0.5 \mu \mathrm{l})$ microinfusion with a characteristic increase in spontaneous MSN frequency and a characteristic decrease in spontaneous FSI frequency. 

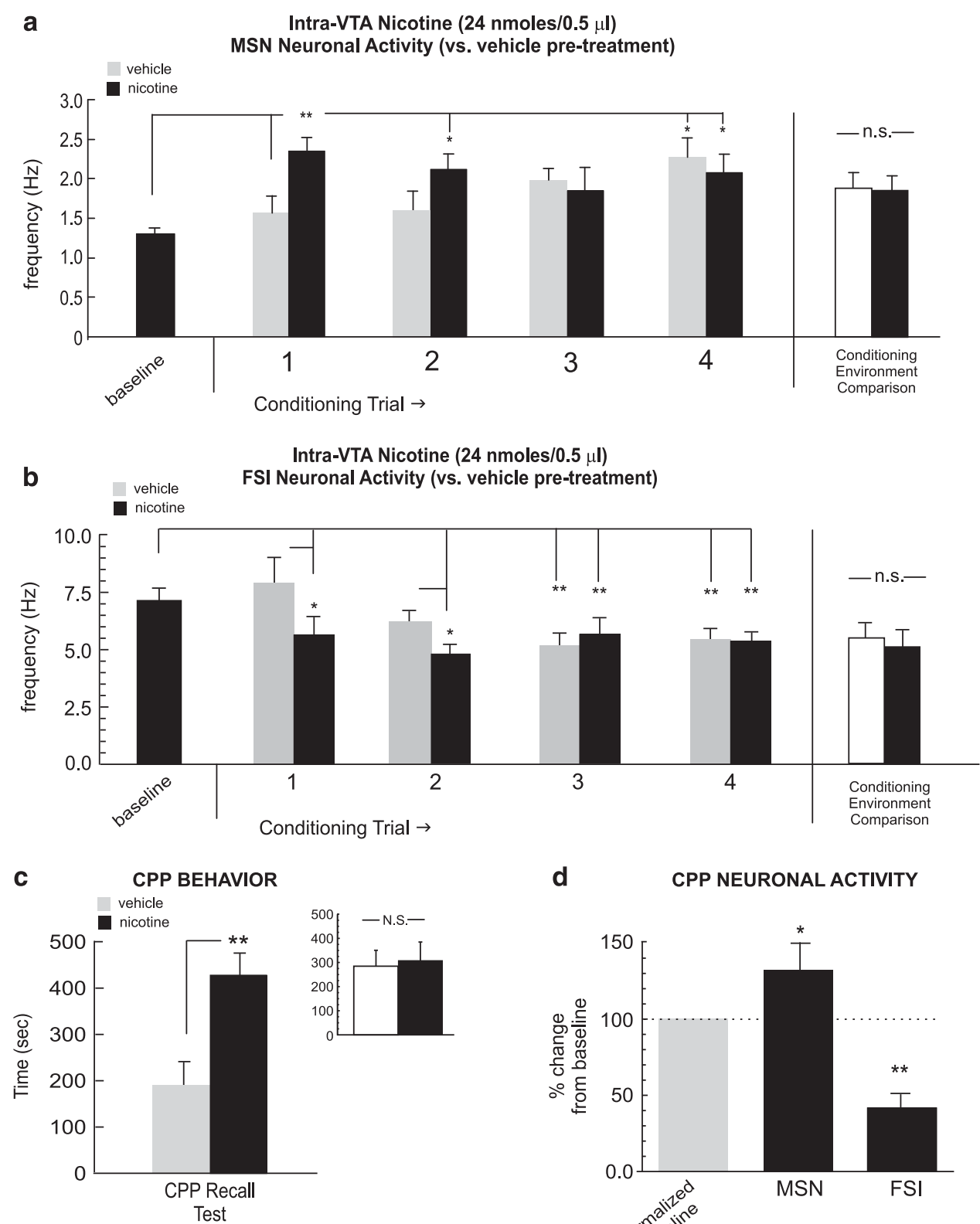

d CPP NEURONAL ACTIVITY
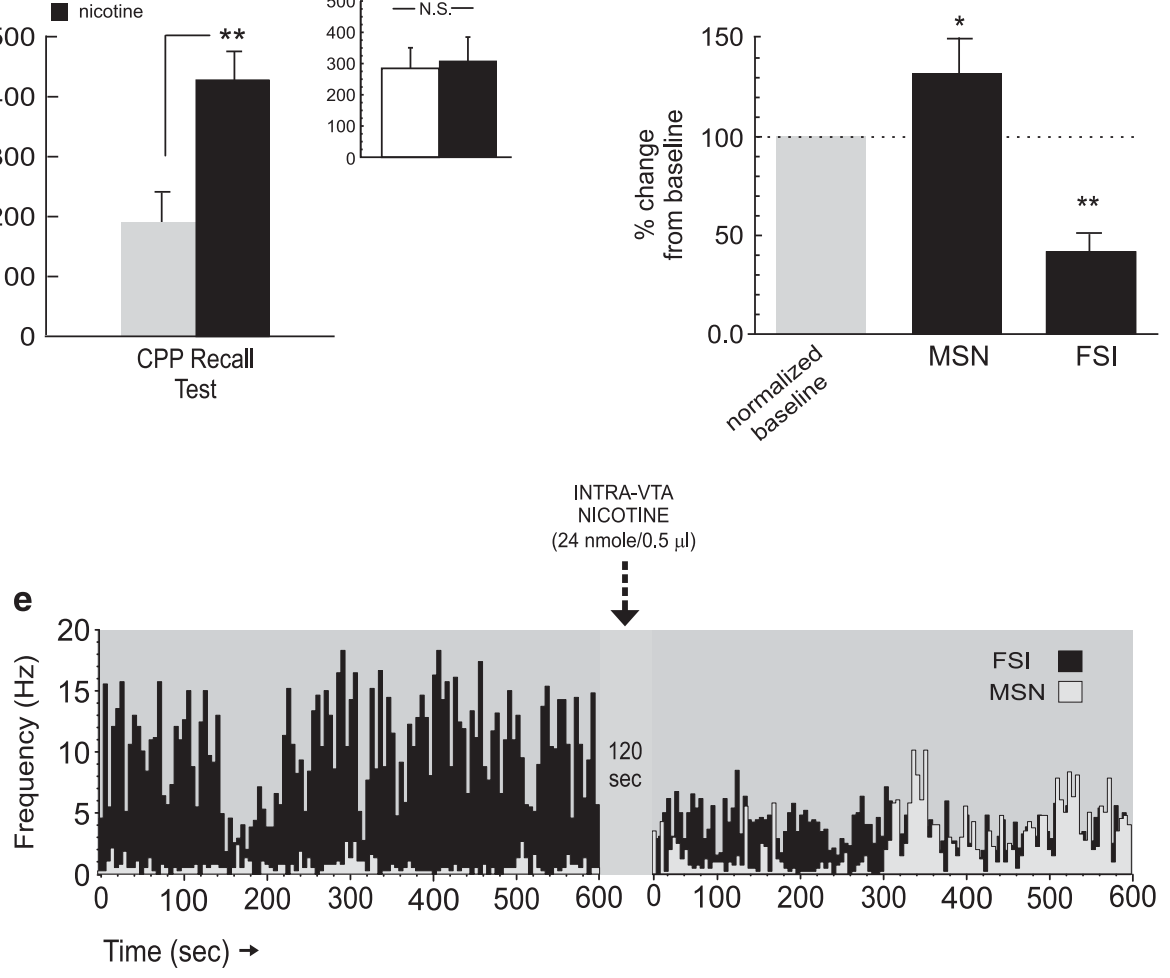
Effects of DA Receptor Blockade on Behavioral and NAshell Neuronal Response Patterns During Nicotine Reward Conditioning

Previous reports have demonstrated that either systemic or intra-NAc blockade of DA transmission has no effect on the rewarding properties of intra-VTA nicotine reward processing (Laviolette and van der Kooy, 2003, 2004, Tan et al, 2009). Accordingly, we next tested the effects of intra-VTA nicotine reward learning $(24 \mathrm{nmol} / 0.5 \mu \mathrm{l})$ following pretreatment with $\alpha$-flu $(0.8 \mathrm{mg} / \mathrm{kg}$; i.p., see Materials and Methods section). Recordings of MSNs $(n=27)$ performed in the home cage (before intra-VTA nicotine conditioning) at either 30 or $150 \mathrm{~min}$ post- $\alpha$-flu administration $(0.8 \mathrm{mg} / \mathrm{kg}$; i.p., see Materials and Methods section) revealed no significant effect on spontaneous activity patterns, relative to baseline (pre-injection) levels (Figure 4a; $\mathrm{F}(2,77)=0.98$; $p>0.05$ ). For MSN recordings during conditioning trials, one-way ANOVA comparing neuronal frequencies during vehicle $v s$ nicotine conditioning sessions revealed a significant effect of conditioning session (vehicle $v s$ nicotine) on firing rates $(\mathrm{F}(8,386)=7.12, p<0.0001)$. Post hoc analysis revealed that MSN firing rates were significantly increased relative to vehicle conditioning levels during the first, second and fourth conditioning trials ( $p$-values $<0.01$ and 0.05 , respectively; Figure 4a) Furthermore, MSN activity levels during nicotine sessions were significantly increased relative to pre-conditioning baseline levels during the first, second, and fourth sessions ( $p$-values $<0.01)$. Comparing MSN neuronal activity patterns across all conditioning sessions recorded in each separate conditioning environment revealed no differences in MSN activity across these environments independently of drug treatment $\left(t_{(26)}=1.42\right.$; $p>0.05$; Figure $4 \mathrm{a}$, far right side). Furthermore, comparing MSN firing activity during pre-conditioning exposures to each conditioning environment alone (see Materials and Methods section) revealed no significant differences between environments $\left(t_{(26)}=0.94 ; p>0.05\right.$; data not shown).

In contrast, recordings of FSIs $(n=24)$ performed in the home cage (before intra-VTA nicotine conditioning) at 30 and $150 \mathrm{~min}$ post- $\alpha$-flu $(0.8 \mathrm{mg} / \mathrm{kg}$; i.p., see Materials and Methods section), revealed a temporally dependent suppression of spontaneous firing activity, relative to baseline (pre-injection) levels $(\mathrm{F}(2,71)=3.9 ; p<0.05$; Figure $4 \mathrm{~b}$ ), with post hoc analysis revealing that $\alpha$-flu pre-treatment significantly decreased spontaneous FSI neuronal activity below baseline levels at both the 30- and 150-min postinjection time point ( $p$-values $<0.01$; Figure $4 \mathrm{~b}$ ). For FSI recordings during conditioning trials, one-way ANOVA comparing frequency rates during vehicle vs nicotine conditioning sessions revealed a significant effect of conditioning session (vehicle $v s$ nicotine) $(\mathrm{F}(8,233)=5.1$, $p<0.0001$ ). Post hoc analysis revealed that FSI firing rates were significantly decreased relative to vehicle conditioning levels during the second, third, and fourth conditioning trials ( $p$-values $<0.01$ and 0.05 , respectively; Figure $4 \mathrm{~b}$ ) Furthermore, FSI activity levels during nicotine sessions were significantly decreased relative to pre-conditioning baseline levels during the second, third, and fourth ( $p$-values $<0.01$ and 0.05 , respectively). Comparing FSI neuronal activity patterns across all conditioning sessions recorded in each separate conditioning environment revealed no differences in FSI activity across these environments independently of drug treatment $\left(t_{(23)}=1.65 ; p>0.05\right.$; Figure $4 \mathrm{~b}$, far right side). Furthermore, comparing FSI firing activity during pre-conditioning exposures to each conditioning environment alone (see Materials and Methods section) revealed no significant differences between environments $\left(t_{(23)}=0.46 ; p>0.05\right.$; data not shown).

Consistent with previous reports (Laviolette and van der Kooy, 2003; Tan et al, 2009), rats pre-treated with $\alpha$-flu before receiving a rewarding dose of intra-VTA nicotine demonstrated a robust CPP at testing $\left(t_{(6)}=3.75 ; p<0.01\right.$; Figure $4 \mathrm{c}$ ), with rats spending significantly more time in the nicotine-paired environment during CPP testing. Post-test analysis comparing times spent in each of the separate conditioning environments (independently of treatment) revealed no significant bias toward either environment $\left(t_{(6)}=1.8 ; p>0.05 ;\right.$ Figure $4 \mathrm{c}$, inset). One-way ANOVA comparing MSN and FSI activity patterns during exposure to either vehicle or nicotine-paired environments during the initial CPP test revealed a significant effect of group on frequency $(\mathrm{F}(2,83)=52.9 ; p<0.0001)$. Post hoc analysis revealed that whereas MSN activity during nicotineenvironment exposure was significantly increased relative to vehicle $(p<0.01)$, FSI activity was significantly suppressed ( $p<0.01$; Figure $4 \mathrm{~d}$ ), revealing a similar MSN/FSI activity pattern divergence during nicotine CPP reward memory recall to the patterns observed during acquisition training. Thus, DA receptor blockade had no effect on the behavioral acquisition of nicotine reward CPP, and this experimental group demonstrated similar patterns of FSI and MSN neuronal activity during the acquisition and recall phases of nicotine reward learning, characterized by increased MSN and decreased FSI firing frequencies.

\section{Neuronal Response Patterns in the NAshell During intra-VTA Nicotine Aversion Conditioning}

Having determined the neuronal patterns associated with the acute rewarding dose of intra-VTA nicotine during CPP

Figure 3 NAshell neuronal group activity patterns during intra-ventral tegmental area (VTA) nicotine reward learning. (a) Neuronal activity patterns during vehicle or nicotine $(24 \mathrm{nmol} / 0.5 \mu \mathrm{l})$ conditioning sessions for isolated medium spiny neurons (MSNs). Total average MSN neuronal activity patterns did not differ across the different conditioning environments, independently of drug treatment (far right side). (b) Neuronal activity patterns during vehicle or nicotine fast-spiking interneuron (FSI) neuronal units. Total average FSI neuronal activity patterns did not differ across the different conditioning environments, independently of drug treatment (far right side). (c) Rewarding behavioral effects of intra-VTA nicotine demonstrated by conditioned place preference (CPP) for nicotine-paired environments during testing. Inset shows average times spent in the separate conditioning environments, independently of treatment condition. (d) Relative to baseline activity, MSNs displayed increased activity and FSls demonstrated inhibited activity during the CPP test. (e) Representative MSN/FSI rastergram overlay, showing 10 min pre intra-VTA nicotine $(24 \mathrm{nmol} / 0.5 \mu \mathrm{l})$ vs post-activity levels with characteristic increased spontaneous firing. In contrast, FSIs typically displayed increased activity following intra-VTA nicotine infusions. Bars represent mean \pm SEM for this and subsequent figures. ${ }^{*} p<0.05 ;{ }^{*} p<0.01$. NAshell, shell region of the NAc; N.S., not significant. 


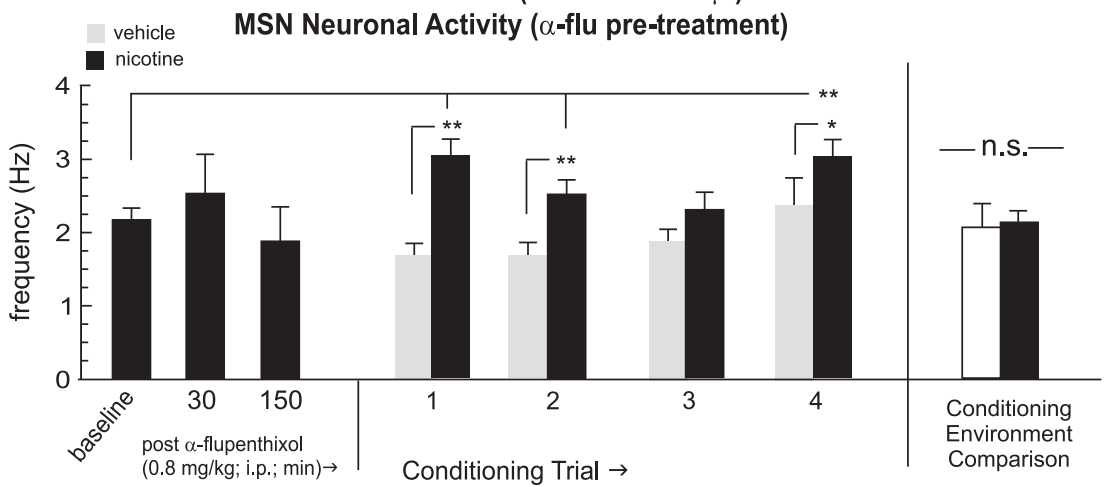

$\begin{array}{ll}\text { b } & \text { vehicle } \\ \text { nicotine } & \text { FSI Neuronal Activity ( } \alpha \text {-flu pre-treatment) }\end{array}$

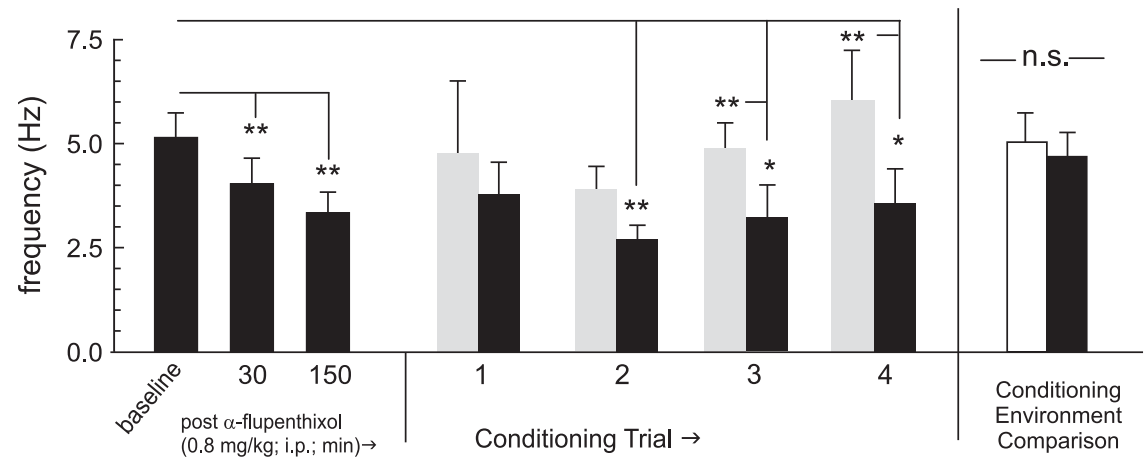

C

CPP BEHAVIOR

d

CPP NEURONAL ACTIVITY
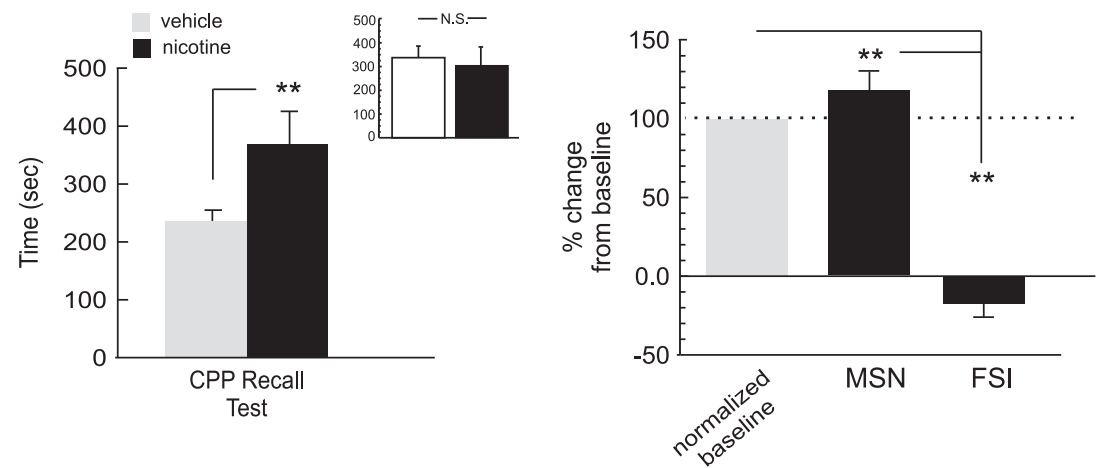

Figure 4 Effects of dopamine (DA) receptor blockade on NAshell neuronal activity patterns during nicotine reward conditioning. (a) Pretreatment with $\alpha$-flupenthixol ( $\alpha$-flu; $0.8 \mathrm{mg} / \mathrm{kg}$; i.p.) has no significant effect on the spontaneous activity levels of NAshell medium spiny neurons (MSNs). However, during conditioning, MSNs show increased activity during the first, second, and fourth nicotine conditioning trials. Total average MSN neuronal activity patterns did not differ across the different conditioning environments, independently of drug treatment (far right side). (b) In contrast, $\alpha$-flu pre-treatment causes a significant attenuation in the spontaneous activity levels of NAshell fast-spiking interneurons (FSIs). However, during conditioning, FSls show decreased activity (relative to vehicle) during the second, third, and fourth nicotine conditioning trials. Total average FSI neuronal activity patterns did not differ across the different conditioning environments, independently of drug treatment (far right side). (c) DA receptor blockade failed to block nicotine reward conditioned place preference (CPP), as rats show a robust nicotine environment preference. Inset shows average times spent in the separate conditioning environments, independently of treatment condition. (d) Relative to baseline activity, MSNs displayed increased activity and FSIs demonstrated inhibited activity during the CPP test. ${ }^{*} p<0.05$; ** $<0.0$ I. NA shell, shell region of the NAc; N.S., not significant.

conditioning, we next examined how NAshell neuronal population activity may be associated with a previously established acutely aversive dose of intra-VTA nicotine $(0.008 \mathrm{nmol} / 0.5 \mu \mathrm{l}$; Laviolette and van der Kooy, 2003, 2004; Tan et al, 2009). For MSN recordings $(n=30)$, one-way ANOVA comparing neuronal frequencies during vehicle $v s$ nicotine conditioning sessions revealed a significant effect of session (vehicle $v s$ nicotine) on firing rates $(\mathrm{F}(8,134)=$ 2.37, $p<0.05)$. Post hoc analysis revealed that MSN firing rates were significantly decreased relative to vehicle levels during the first, second, and fourth conditioning trials ( $p$-values <0.05; Figure 5a) Furthermore, MSN activity levels during nicotine sessions were significantly decreased relative to baseline levels during the first, second, and 

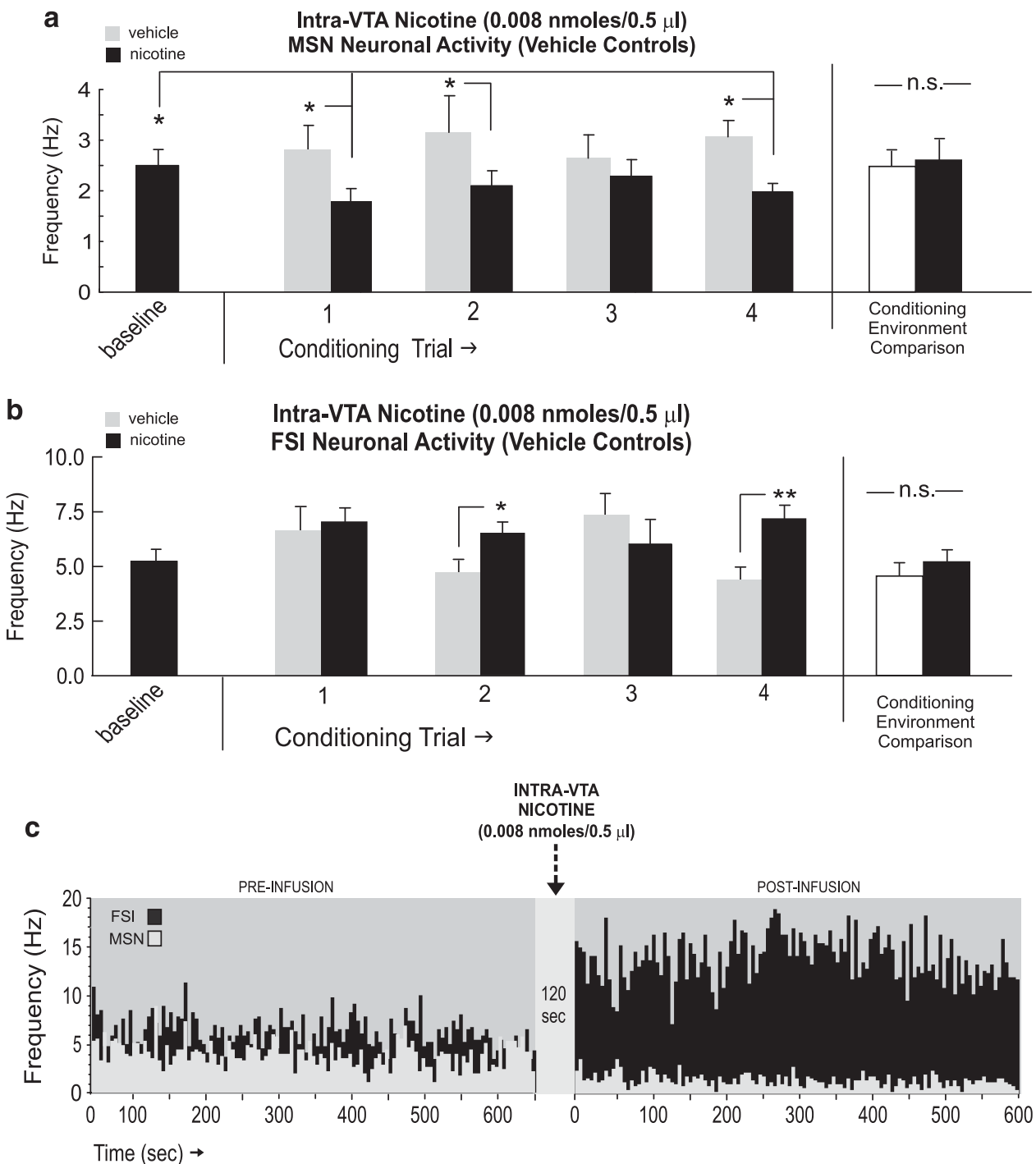

Figure 5 NAshell neuronal activity patterns during intra-ventral tegmental area (VTA) nicotine aversion learning. (a) Neuronal activity patterns during vehicle or nicotine $(0.008 \mathrm{nmol} / 0.5 \mu \mathrm{l})$ conditioning sessions for isolated medium spiny neuron (MSN) neuronal units demonstrating increased activity during the first, second, and fourth conditioning trials. Total average MSN neuronal activity patterns did not differ across the different conditioning environments, independently of drug treatment (far right side). (b) Fast-spiking interneuron (FSI) neuronal units recorded during conditioning sessions revealed increased activity relative to vehicle conditioning on the second and fourth trials. Total average FSI neuronal activity patterns did not differ across the different conditioning environments, independently of drug treatment (far right side). (c) Representative MSN/FSI neuronal rastergram overlay showing I0 min pre intra-VTA nicotine $(0.008 \mathrm{nmol} / 0.5 \mu \mathrm{l})$ vs post-activity levels with characteristic decreased spontaneous MSN firing vs increased FSI neuronal firing rates. * $p<0.05$; *** $<0.01$. NAshell, shell region of the NAc; N.S., not significant.

fourth sessions ( $p$-values $<0.05$ ). Intra-VTA vehicle did not induce any significant change from baseline levels during any conditioning trials ( $p$-values $>0.05$ ). Comparing MSN neuronal activity patterns across all conditioning sessions recorded in each separate conditioning environment revealed no differences in MSN activity across these environments independently of drug treatment $\left(t_{(29)}=1.93 ; p>0.05\right.$; Figure $5 \mathrm{a}$, far right side). Furthermore, comparing MSN firing activity during pre-conditioning exposures to each conditioning environment alone (see Materials and Methods section) revealed no significant differences between environments $\left(t_{(29)}=1.14 ; p>0.05\right.$; data not shown).

For FSI recordings $(n=22)$, one-way ANOVA comparing neuronal frequencies during vehicle $v s$ nicotine conditioning sessions revealed a significant effect of conditioning session (vehicle $v$ s nicotine) on firing rates $(\mathrm{F}(8,373)=79.4$, $p<0.0001$ ). Post hoc analysis revealed that FSI firing rates were significantly increased relative to vehicle levels during the second and fourth conditioning trials ( $p$-values $<0.05$ and 0.01 , respectively; Figure $5 b$ ). However, FSI activity levels did not increase significantly above baseline recording levels during either nicotine or vehicle sessions across conditioning trials ( $p$-values $>0.05)$. Comparing FSI neuronal activity patterns across all conditioning sessions recorded in each separate conditioning environment revealed no differences in MSN activity across these environments independently of drug treatment $\left(t_{(21)}=1.15 ; p>0.05\right.$; Figure $5 \mathrm{~b}$, far right side). Furthermore, comparing FSI firing activity during pre-conditioning exposures to each conditioning environment alone (see Materials and 


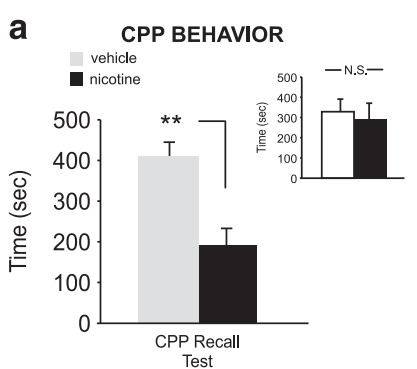

b CPP NEURONAL ACTIVITY
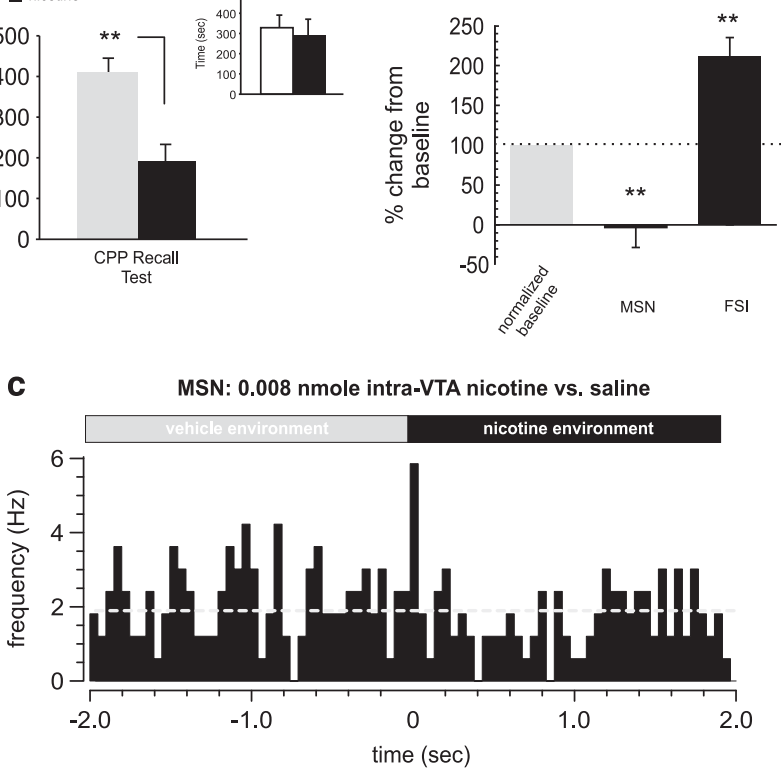

d

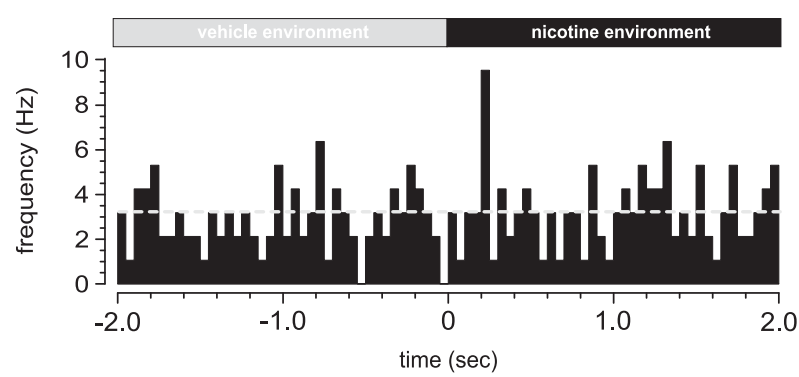

Figure 6 Behavioral and neuronal responses to nicotine aversion conditioning. (a) A lower dose of intra-ventral tegmental area (VTA) nicotine $(0.008 \mathrm{nmol} / 0.5 \mu \mathrm{l})$ produces a strong aversion to nicotine-paired environments. Inset shows average times spent in the separate conditioning environments, independently of treatment condition. (b) During place conditioning testing, medium spiny neurons (MSNs) show decreased activity relative to baseline and fast-spiking interneurons (FSIs) show increased activity, during exposure to nicotine-paired environments. (c) Representative MSN recorded during the place conditioning test, showing a characteristic decrease in spontaneous activity during nicotine-environment exposure. (d) In contrast, a typical FSI neuronal activity raster showing a characteristic increase in activity during nicotine-environmental exposure. ${ }^{*} p<0.05$; *** $p<0.0$ I. N.S., not significant.

Methods section) revealed no significant differences between environments $\left(t_{(21)}=0.002 ; p>0.05\right.$; data not shown). In Figure $5 c$, we present sample MSN and FSI overlaid recording traces showing $10 \mathrm{~min}$ baseline activity epoch followed by a 10-min post-infusion recording following intra-VTA nicotine $(0.008 \mathrm{nmol} / 0.5 \mu \mathrm{l})$ microinfusion with a characteristic decrease in spontaneous MSN frequency and a characteristic increase in spontaneous FSI frequency.

Consistent with previous reports (Laviolette and van der Kooy, 2003; Tan et al, 2009), this dose of intra-VTA nicotine $(0.008 \mathrm{nmol} / 0.5 \mu \mathrm{l})$ produced a robust behavioral CPA $\left(t_{(7)}=2.63 ; p<0.05\right.$; Figure $\left.6 \mathrm{a}\right)$, with rats spending significantly less time in the nicotine-paired environment during CPP testing. Post-test analysis comparing times spent in each of the separate conditioning environments (independently of treatment) revealed no significant bias toward either environment $\left(t_{(7)}=0.28 ; p>0.05\right.$; Figure 6a, inset). One-way ANOVA comparing MSN and FSI activity patterns during exposure to either vehicle or nicotinepaired environments during the initial CPP test (rats tested in a drug-free state, see Materials and Methods section) revealed a significant effect of group on firing frequency $(\mathrm{F}(2,35)=8.22 ; p<0.01)$ with post hoc analysis revealing that whereas MSN activity during nicotine-environment exposure was significantly decreased relative to baseline $(p<0.01)$, FSI activity was significantly increased $(p<0.01$; Figure $6 \mathrm{~b}$ ), revealing a similar MSN/FSI activity pattern divergence during nicotine $\mathrm{CPP}$ reward memory recall to the patterns observed during acquisition training (Figure 4). In Figure $6 \mathrm{c}$ and d, we present samples of typical NAshell MSN vs FSI spontaneous activity patterns recorded during transitions ( $2 \mathrm{~s}$ pre and post) between vehicle $v s$ nicotinepaired environments during the CPP test, showing a typical transient decrease in MSN neuronal activity during nicotine-environment exposure (Figure 6c) and a typical increase in FSI activity during the transition to the nicotinepaired environment (Figure 6d).

\section{DA Receptor Blockade Reverses Behavioral and NAshell Neuronal Response Patterns During Nicotine Aversion Learning Acquisition}

Previous reports have demonstrated that blockade of DA transmission induces a behavioral switch in the conditioning effects of either intra-VTA or systemic nicotine, from aversion to reward (Laviolette and van der Kooy, 2003, 2004, Tan et al, 2009). Accordingly, we next tested the effects of intra-VTA nicotine aversion learning following pre-treatment with $\alpha$-flu $(0.8 \mathrm{mg} / \mathrm{kg}$; i.p.; see Materials and Methods section). Recordings of MSNs $(n=27)$ performed in the home cage (before intra-VTA nicotine conditioning) at either 30 or $150 \mathrm{~min}$ post- $\alpha$-flu $(0.8 \mathrm{mg} / \mathrm{kg}$; i.p.; see Materials and Methods section) administration revealed no significant effect on spontaneous activity patterns, relative to baseline (pre-injection) levels $(\mathrm{F}(27,77)=0.07 ; p>0.05$; Figure 7a). For MSN recordings during conditioning trials, one-way ANOVA comparing neuronal frequencies during vehicle $v s$ nicotine conditioning sessions revealed a significant effect of conditioning session (vehicle $v s$ nicotine) on firing rates $(\mathrm{F}(8,146)=47.9 ; p<0.0001)$. Post hoc analysis revealed that MSN firing rates were significantly increased relative to vehicle levels during the first, second, and fourth conditioning trials ( $p$-values $<0.01$ and 0.05 , respectively Figure 7a). Furthermore, MSN activity levels during nicotine sessions were significantly increased relative to baseline levels during the first, second, and fourth sessions ( $p$-values <0.05). Comparing MSN neuronal activity patterns across all conditioning sessions recorded in each separate conditioning environment revealed no differences in MSN activity across these environments independently of drug treatment $\left(t_{(26)}=0.07 ; p>0.05\right.$; Figure $7 \mathrm{a}$, far right side). Furthermore, comparing MSN firing activity during pre-conditioning exposures to each conditioning environment alone (see Materials and Methods section) revealed no 
a

vehicle

Intra-VTA Nicotine (0.008 nmoles/0.5 $\mu$ l)

nicotine

MSN Neuronal Activity ( $\alpha$-flu pre-treatment)

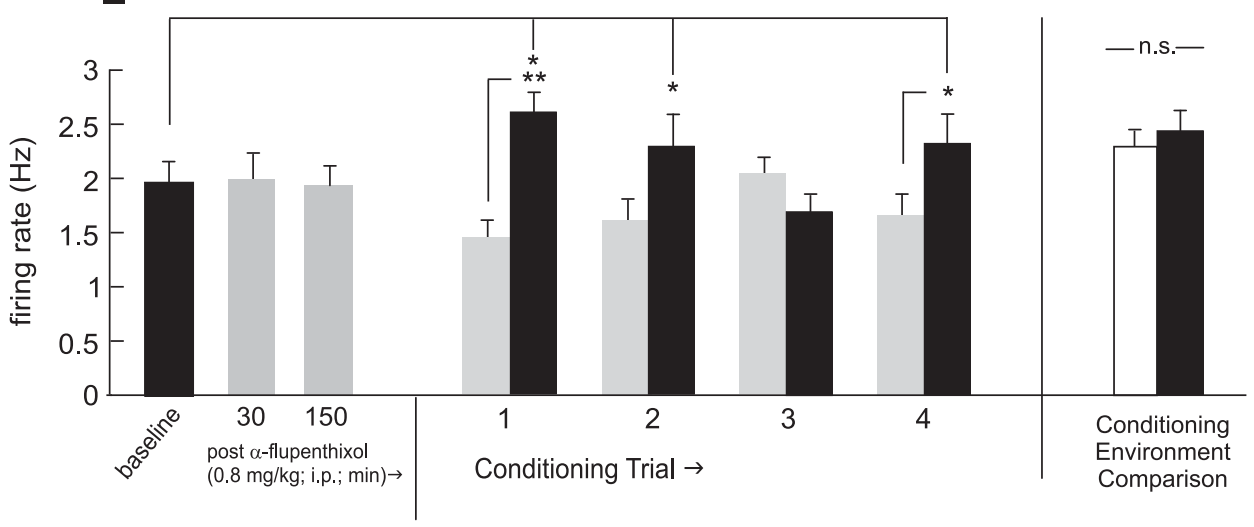

b

vehicle Intra-VTA Nicotine $(0.008 \mathrm{nmoles} / 0.5 \mu \mathrm{l})$

nicotine FSI Neuronal Activity ( $\alpha$-flu pre-treatment)
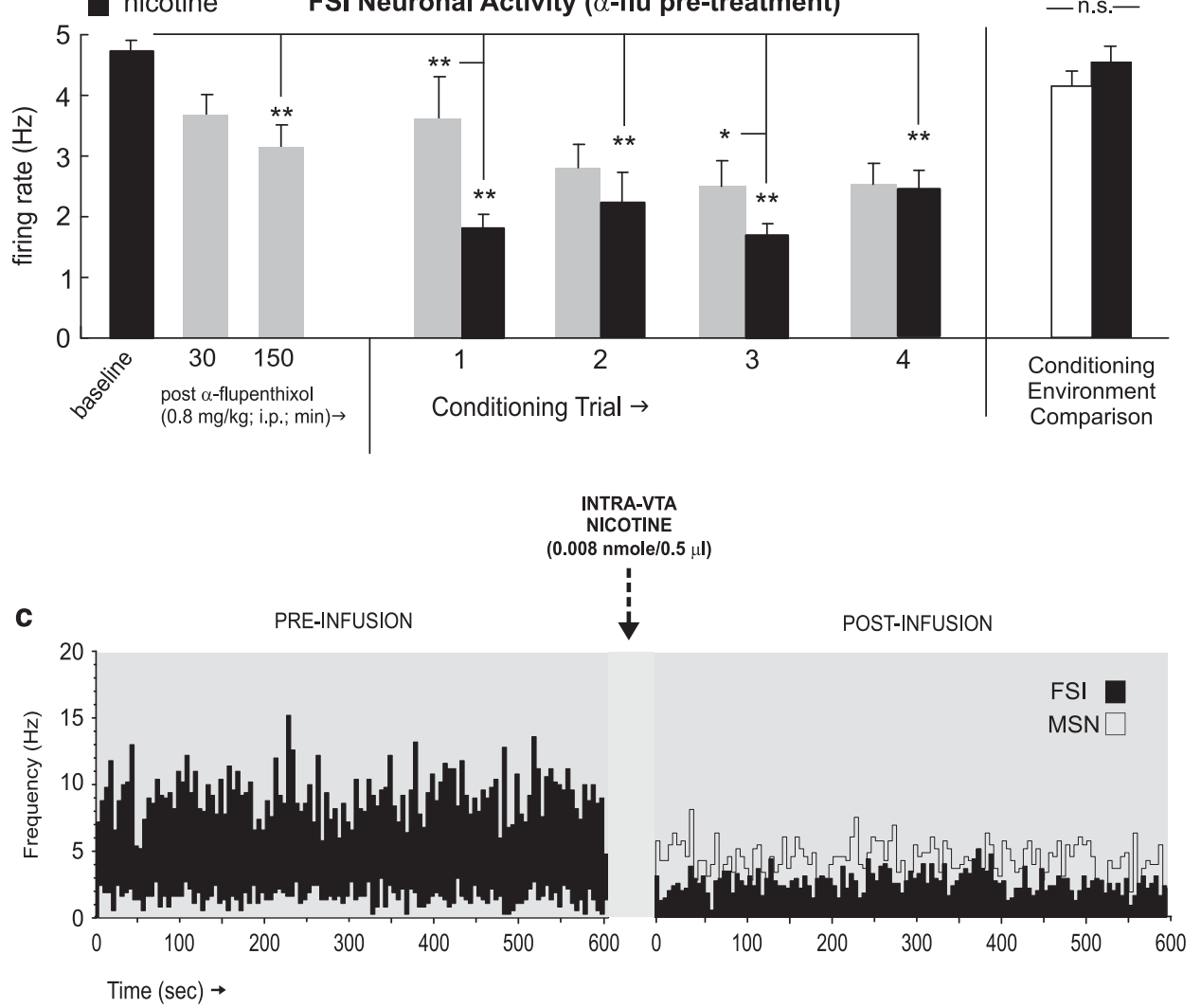

Figure 7 Effects of dopamine receptor blockade on NAshell neuronal activity patterns during nicotine aversion conditioning. (a) Pretreatment with $\alpha$-flupenthixol ( $\alpha$-flu; $0.8 \mathrm{mg} / \mathrm{kg}$; i.p.) has no significant effect on the spontaneous activity levels of NAshell medium spiny neurons (MSNs). However, MSNs show increased activity during the first, second, and fourth nicotine conditioning trials. Total average MSN neuronal activity patterns did not differ across the different conditioning environments, independently of drug treatment (far right side). (b) In contrast, $\alpha$-flu pre-treatment causes a significant attenuation in the spontaneous activity levels of NAshell fast-spiking interneurons (FSIs). During conditioning, FSIs show decreased activity (relative to vehicle) during the first, second, and third nicotine conditioning trials. Total average FSI neuronal activity patterns did not differ across the different conditioning environments, independently of drug treatment (far right side). (c) Representative MSN/FSI neuronal rastergram overlay showing 10 min pre intra-ventral tegmental area nicotine $(0.008 \mathrm{nmol} / 0.5 \mu \mathrm{l})$ vs post-activity levels with characteristic increased spontaneous MSN vs decreased FSI firing rates in the presence of $\alpha$-flu. $* p<0.05 ;{ }^{*} * 0<0.01$. NA shell, shell region of the NAc; N.S., not significant.

significant differences between environments $\left(t_{(26)}=0.79\right.$; $p>0.05$; data not shown).

In contrast, recordings of FSIs $(n=15)$ performed in the home cage (before intra-VTA nicotine conditioning) at either 30 or $150 \mathrm{~min}$ post $-\alpha$-flu $(0.8 \mathrm{mg} / \mathrm{kg}$; i.p., see Materials and Methods section) revealed a temporally dependent suppression of spontaneous firing activity, relative to baseline (pre-injection) levels $(\mathrm{F}(25,77)=3.86 ; p>0.05$; 
Figure 7b), with post hoc analysis revealing that $\alpha$-flu pretreatment significantly decreased FSI neuronal activity below baseline levels at the 150 -min post-injection time point $(p<0.01$; Figure $7 b)$. For FSI recordings during conditioning trials, one-way ANOVA comparing frequency rates during vehicle $v s$ nicotine conditioning sessions revealed a significant effect of conditioning session (vehicle $v s$ nicotine $)(\mathrm{F}(8,147)=17.02 ; p<0.0001)$. Post hoc analysis revealed that FSI firing rates were significantly decreased relative to vehicle levels during the first and third conditioning trials ( $p$-values $<0.01$ and 0.05 , respectively; Figure 7b) Furthermore, FSI activity levels during nicotine sessions were significantly decreased relative to baseline levels during all four conditioning trials ( $p$-values $<0.01$ ), and during vehicle sessions, during the second to fourth trials ( $p$-values $<0.01)$. Comparing FSI neuronal activity patterns across all conditioning sessions recorded in each separate conditioning environment revealed no differences in MSN activity across these environments independently of drug treatment $\left(t_{(14)}=1.15 ; p>0.05\right.$; Figure $7 \mathrm{~b}$, far right side). Furthermore, comparing FSI firing activity during pre-conditioning exposures to each conditioning environment alone (see Materials and Methods section) revealed no significant differences between environments $\left(t_{(14)}=1.23\right.$; $p>0.05$; data not shown).

In Figure $7 \mathrm{c}$, we present sample MSN and FSI overlaid recording traces in an $\alpha$-flu pre-treated rat, showing $10 \mathrm{~min}$ baseline activity epoch followed by a 10-min post-infusion recording following intra-VTA nicotine $(0.008 \mathrm{nmol} / 0.5 \mu \mathrm{l})$. A characteristic increase in spontaneous MSN frequency and a characteristic decrease in spontaneous FSI frequency are demonstrated, similar to results obtained with the higher, acutely rewarding dose of intra-VTA nicotine (Figure 3e).

Behaviorally, rats pre-treated with $\alpha$-flu demonstrated a robust CPP at testing $\left(t_{(7)}=3.34 ; p<0.01\right.$; Figure $\left.8 \mathrm{a}\right)$, with rats spending significantly more time in the nicotine-paired environment during CPP testing. Post-test analysis comparing times spent in each of the separate conditioning environments (independently of treatment) revealed no significant bias toward either environment $\left(t_{(7)}=0.04\right.$; $p>0.05$; Figure $8 \mathrm{a}$, inset). One-way ANOVA comparing MSN and FSI activity patterns during exposure to either vehicle or nicotine-paired environments during the initial CPP test revealed a significant effect of group on frequency $(\mathrm{F}(2,38)=243.9 ; p<0.0001)$. Post hoc analysis revealed that whereas MSN activity during nicotine-environment exposure was significantly increased relative to vehicle $(p<0.01)$, FSI activity was significantly suppressed $(p<0.01$; Figure $8 \mathrm{~b})$, revealing a similar MSN/FSI activity pattern divergence during nicotine CPP reward memory recall to the patterns observed during acquisition training. In Figure 8c and d, we present samples of typical NAshell MSN $v s$ FSI spontaneous activity patterns, recorded during transitions ( $2 \mathrm{~s}$ pre and post) between vehicle $v s$ nicotinepaired environments during the CPP test, showing a typical transient increase in MSN neuronal activity during nicotine-environment exposure (Figure 8c) and a typical decrease in FSI activity during the transition to the nicotinepaired environment (Figure 8d).

In Figure 9, we present a schematic summary comparison of MSN vs FSI firing rates during nicotine behavioral
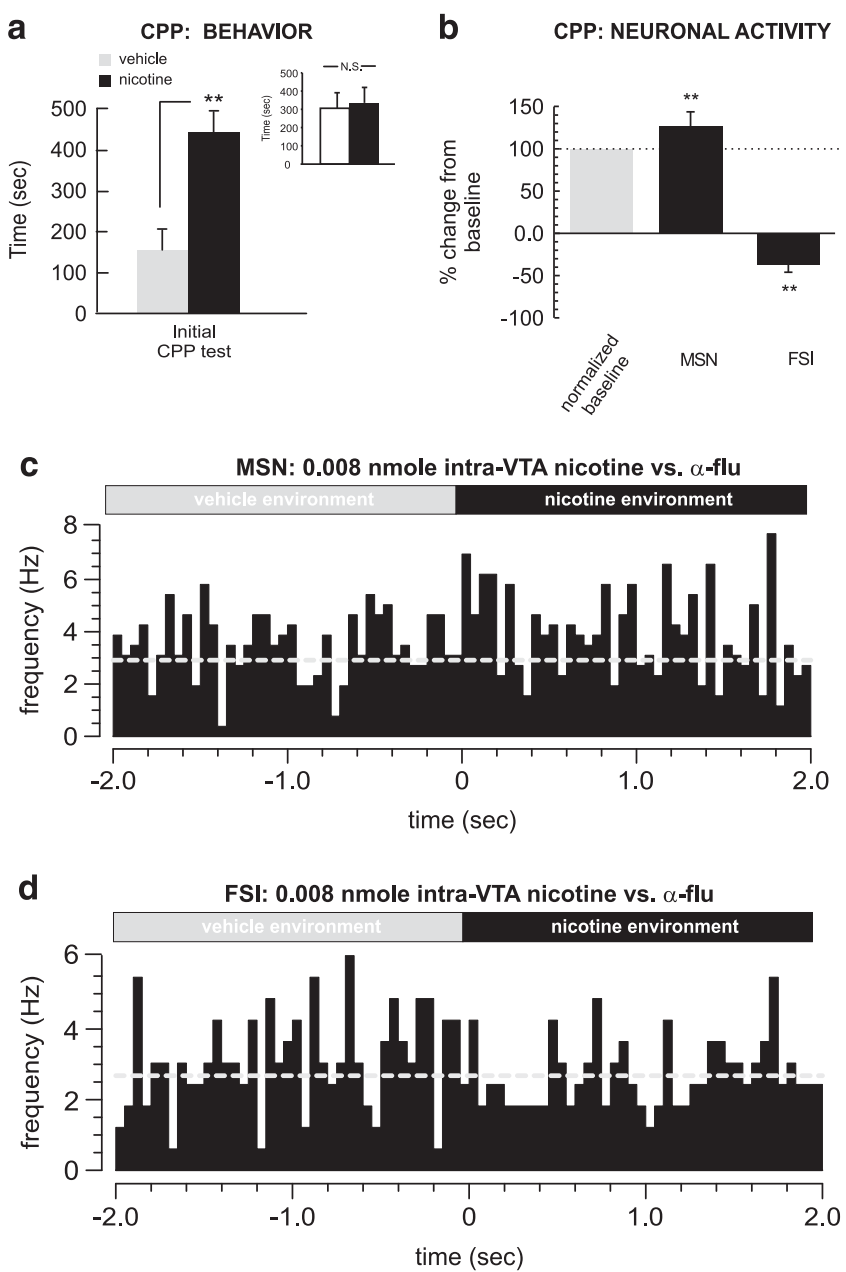

Figure 8 Behavioral and neuronal effects of dopamine receptor blockade during nicotine aversion conditioning. (a) Pretreatment with $\alpha$-flupenthixol $(0.8 \mathrm{mg} / \mathrm{kg}$; i.p.) switches a normally aversive dose of intraventral tegmental area nicotine $(0.008 \mathrm{nmol} / 0.5 \mu \mathrm{l})$ into a robust conditioned place preference (CPP). Inset shows average times spent in the separate conditioning environments, independently of treatment condition. (b) During CPP testing, medium spiny neurons (MSNs) show increased activity relative to baseline and fast-spiking interneurons (FSIs) show decreased activity, during exposure to nicotine-paired environments. (c) Representative MSN recorded during testing, showing a characteristic increase in spontaneous activity during nicotine-environment exposure. (d) In contrast, a typical FSI neuronal activity raster showing a characteristic decrease in activity during nicotine-environmental exposure. $* p<0.05$; *** $<0.0$ I. N.S., not significant.

conditioning sessions for the acutely rewarding dose of intra-VTA nicotine $(24 \mathrm{nmol} / 0.5 \mu \mathrm{l}$; Figure 9a); an acutely rewarding dose of intra-VTA nicotine $(24 \mathrm{nmol} / 0.5 \mu \mathrm{l})$ following systemic $\alpha$-flu (Figure 9b); an acutely aversive dose following vehicle pre-treatment $(0.008 \mathrm{nmol} / 0.5 \mu \mathrm{l}$; Figure 9c) and the behaviorally 'switched' dose (aversion to reward, $0.008 \mathrm{nmol} / 0.5 \mu \mathrm{l}$ ) following pre-treatment with systemic $\alpha$-flu (Figure 9d).

\section{DISCUSSION}

Identifying the underlying neural mechanisms controlling how nicotine's bivalent rewarding or aversive stimulus 
a

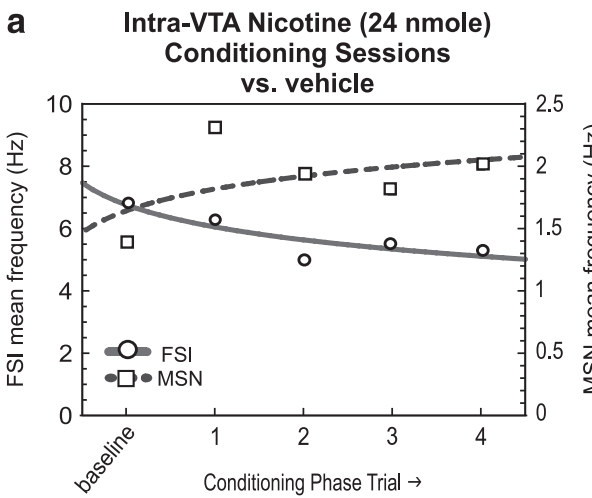

c

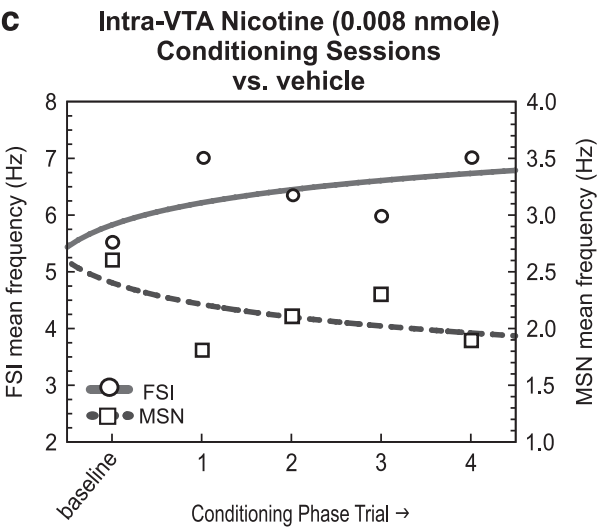

b

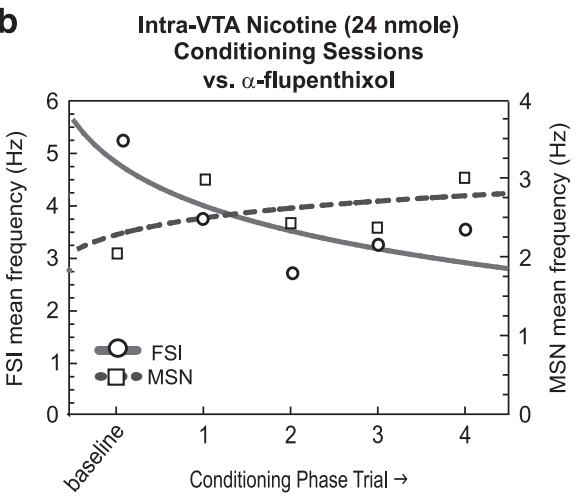

d

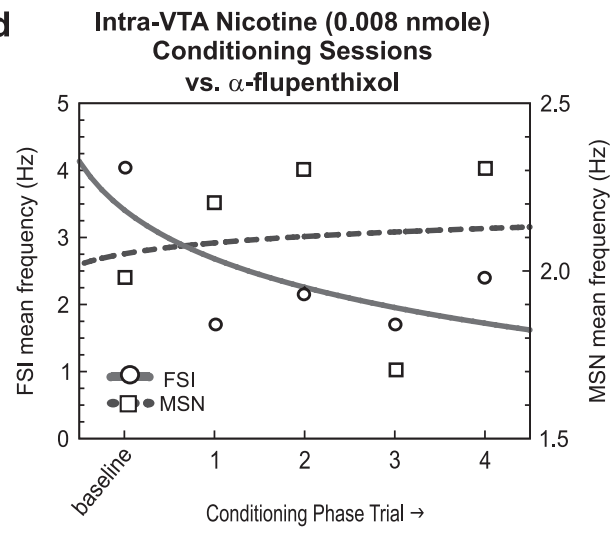

Figure 9 Summary of NAshell fast-spiking interneuron (FSI) vs medium spiny neuron (MSN) activity patterns during nicotine conditioning sessions. (a) Acutely rewarding intra-ventral tegmental area (VTA) nicotine $(24 \mathrm{nmol} / 0.5 \mu \mathrm{l})$ evokes increased MSN and decreased FSI activity over conditioning trials, while (b) challenge of this dose of intra-VTA nicotine with $\alpha$-flupenthixol ( $\alpha$-flu) pre-treatment does not alter this activity pattern. (c) In contrast, an acutely aversive dose of intra-VTA nicotine $(0.008 \mathrm{nmol} / 0.5 \mu \mathrm{l})$ produces the opposite pattern of activity with decreased MSN activity and increased FSI activity. (d) However, pre-treatment with $\alpha$-flu $(0.8 \mathrm{mg} / \mathrm{kg}$; i.p.) switches a normally 'aversive' nicotine-related neuronal conditioning pattern into one similar to the acutely rewarding dose of intra-VTA nicotine. NAshell, shell region of the NAc.

properties are processed is critical to understanding the dependence-producing effects of nicotine, particularly given that relative sensitivity to these early effects are critical determinants of the development and persistence of tobacco addiction (Perkins, 1995; Pomerleau, 1995). The present findings provide evidence for distinct neuronal activity signatures in the NAshell associated either with rewarding or aversive nicotine-related behavioral effects. Although reward-related nicotine conditioning was associated with increased MSN activity and decreased FSI activity, aversive effects of nicotine were associated with the opposite pattern of NAshell neuronal activity, both during acquisition and recall of nicotine-related associative memories. Remarkably, systemic blockade of DA transmission switched nicotine aversions into reward, concomitant with a switch in NAshell neuronal activity patterns and, specifically, an acute inhibition of NAshell FSI neuronal activity. These activity patterns were observed not only following intra-VTA reward or aversion conditioning, but during the drug-free, acute CPP recall testing phase in the absence of intra-VTA nicotine.

Although the precise role of the ventral striatum in processing rewarding or aversive motivational information is not known, considerable evidence implicates a role for differential MSN and FSI neuronal activity patterns. Thus, natural or psychostimulant conditioned rewarding stimuli can activate MSN neuronal populations recorded in rodents during behavioral reinforcement paradigms (Lansink et al, 2010; Ma et al, 2013). Indeed, Taha and Fields (2005) reported that a plurality of identified MSNs showed excitatory responses associated with sucrose reward exposure in rats. In primates, striatal interneurons have been reported to show spontaneous inhibitory responses in response to conditioned reinforcers (Joshua et al, 2008; Kimura et al, 1984), suggesting that spontaneous inhibition of NAc FSIs may be a functional correlate of behavioral reward processing. Interestingly, a study from Wiltschko et al (2010) reported that systemic administration of high doses of a D2 receptor antagonist, eticlopride, caused strong inhibition in the spontaneous activity rates of NAc FSI populations recorded in rats, consistent with the present results. Furthermore, we have reported previously that specific blockade of D2 receptors within the shell (but not core) division of the NAc is sufficient to switch nicotine aversion behaviors into rewarding effects (Laviolette et al, 2008).

Nicotine strongly evokes DA release in the NAc (Pontieri et al, 1996; Zocchi et al, 2003), and DA transmission via D2 or D1 receptors potently modulates the motivational properties of either systemic or intra-VTA nicotine directly 
within the NAc (Laviolette et al, 2008; Laviolette and van der Kooy, 2003). Whereas the acute, aversive properties of nicotine are DA dependent, the acute rewarding properties of nicotine can be mediated via substrates extrinsic to the mesolimbic system, such as the pedunculopontine tegmental nucleus (Corrigall et al, 2001; Laviolette et al, 2002). Furthermore, pharmacological or lesioninduced blockade of the mesolimbic DA system strongly potentiates the rewarding properties of both systemic and intra-VTA nicotine (Laviolette et al, 2008; Laviolette and van der Kooy, 2003; Sellings et al, 2008; Tan et al, 2009). Nevertheless, the neuronal mechanisms underlying these phenomena have not previously been characterized. Given that the acute aversive properties of intra-VTA nicotine require mesolimbic DA transmission (Laviolette et al, 2008; Laviolette and van der Kooy, 2003; Sellings et al, 2008; Tan et al, 2009), the NAshell neuronal activity patterns observed with a behaviorally aversive dose of intra-VTA nicotine may be related to the actions of DA within the NAc. For example, in vitro reports have demonstrated that DA may increase activity of FSI populations via direct or indirect synaptic influences. Furthermore, D1 receptor agonists have been shown to excite GABAergic FSIs in vitro (Aosaki et al, 1998; Bracci et al, 2002; Pisani et al, 2000). In contrast, the effects of DA on MSN populations have been reported to be excitatory when acting via D1 receptors and $\mathrm{K}^{+}$channel inhibition (Podda et al, 2010), or inhibitory when acting via D2 receptor substrates (Perez et al, 2006). In the context of the present findings, it is of interest that during in vivo iontophoretic investigations, lower concentrations of DA have been reported to facilitate excitatory, glutamate-dependent NAc neuronal activity, whereas higher doses are predominantly inhibitory (Berger et al, 1987; Hu et al, 1990; Hu and White, 1997). Although these studies do not differentiate between FSI and MSN neuronal populations specifically (and were performed under anesthesia), they demonstrate a bi-phasic effect of DAergic tone on spontaneous neuronal population activity within the NAc.

Assuming the possibility of direct VTA actions on NAc MSNs, it is possible that lower concentrations of intra-VTA nicotine (which would presumably result in relatively lower levels of intra-NAc DA release) may directly excite MSNs via a similar mechanism. In contrast, the higher, rewarding dose of nicotine (which may result in higher NAshell DA release) was associated with reduced FSI activity in the NAshell; an effect that was mimicked by DA antagonist pretreatment, suggesting that nicotine-related VTA $\rightarrow$ NAshell reward signals may operate via direct functional inhibition of FSIs and a concomitant increase in MSN activity. Such an indirect mechanism is further suggested by the finding that DA receptor blockade appeared to have no acute effect on NAshell MSN activity, and increases in MSN activity were only observable following intra-VTA nicotine administration and during the acute CPP recall test. However, consistent with previous reports (Laviolette and van der Kooy, 2003; Tan et al, 2009), DA receptor blockade failed to behaviorally block nicotine reward and produced no alterations in overall FSI/MSN response patterns, consistent with the lack of a behavioral alteration. This would suggest that even if higher doses of intra-VTA nicotine may induce greater DA release levels, neither the behaviorally rewarding nor neuronal patterns associated with intra-VTA nicotine reward processing are dependent upon DA transmission.

The NAshell mediates the rewarding properties of various drugs of abuse, including nicotine (Mereu et al, 1987; Picciotto and Corrigall, 2002). Following systemic administration of a single dose of nicotine, there is a greater increase in DA overflow in NAshell than core, an effect thought to underlie nicotine-related motivational effects (Cadoni et al, 2009; Nisell et al, 1994; Pontieri et al, 1996). Further, The NAc contains populations of $\gamma$-aminobutyric acid (GABA)-containing and cholinergic interneurons, (Smith and Bolam, 1990). In the present study, we observed increased group activity in FSI populations, concomitant with decreased spontaneous activity of presumed MSN populations during nicotine aversion conditioning. Such a relationship is consistent with previous evidence demonstrating that GABAergic FSI's form perisomatic synapses with associated MSNs, which in turn can provide powerful feedforward inhibition on MSNs (Tepper et al, 2010; Planert et al, 2010). Nevertheless, while opposing activity patterns were observed during CPP recall testing, we did not observe consistent inhibitory $v s$ excitatory effects in terms of relative FSI and MSN activity patterns across all aversive or rewarding intra-VTA nicotine conditioning trials. This may be related to the neurons being sampled during a specific recording session, which may reflect a more complex interrelationship between nicotine-modulation of respective FSI or MSN neuronal populations.

It is of interest that during some acquisition trials for both rewarding and aversive nicotine-related conditioning, we observed relative increases or decreases in FSI/MSN populations that appeared to generalize to both vehicle and nicotine training sessions. One possibility is that these responses represented the effects of a common learning mechanism that generalized across both conditioning environments during the course of training. Although future studies are required to more clearly identify these potential learning mechanisms, non-pharmacological conditioning mechanisms are known to be associated with nicotine-related learning and behavioral conditioning (Caggiula et al, 2002; Chaudhri et al, 2006). Although in the present protocol, vehicle and nicotine conditioning sessions took place on separate days, other conditioning cues associated generally with the behavioral protocol (eg, the microinfusion procedure and experimental handling) may have served to induce generalized conditioned responses, reflected in common directionality of NAc neuronal responses across trials.

These results are consistent with prior evidence demonstrating that the aversive effects of nicotine are mediated via mesolimbic DA transmission (Laviolette et al, 2008; Laviolette and van der Kooy 2003; Sellings et al, 2008; Tan et al, 2009). Blockade of DA transmission reversed the behaviorally aversive properties of nicotine, switching nicotine CPA into a robust CPP. This behavioral switch was correlated with inhibitory effects on FSIs during the conditioning phase. Acutely, DA receptor antagonism caused a profound inhibition on NAshell FSI activity, consistent with NAc neuronal activity parameters observed during the acquisition of a normally highly rewarding dose of intra-VTA nicotine. This effect was similarly observed during nicotine reward learning to a higher dose of intra-VTA nicotine, in 
the absence of DA receptor blockade, and led to a behavioral and neuronal pattern switch in the valence of nicotine from aversion to reward, suggesting that blockade of DA transmission is sufficient to induce a reward-related neuronal activity signature in the presence of a normally aversive dose of intra-VTA nicotine. Although the current studies do not discriminate between GABAergic vs cholinergic interneuron populations within the NAc, an interesting report from Threlfell et al (2012) demonstrated that in vitro optogenetic activation of intra-accumbens cholinergic interneurons was sufficient to induce the pre-synaptic release of DA from presumptive VTA terminals. This mechanism would be consistent with the present findings wherein the acute, aversive effects of nicotine were associated with increased FSI neuronal activity and with previous evidence, demonstrating that the behaviorally aversive properties of nicotine are mediated through DAdependent mechanisms directly in the NAc (Laviolette et al, 2008; Laviolette and van der Kooy, 2003; Tan et al, 2009).

An important caveat to the present findings is that the behavioral effects of nicotine were limited to an examination of intra-VTA nicotine administration, focusing on previously established conditioning doses known to produce either aversive or rewarding conditioning effects (Laviolette et al, 2008; Laviolette and van der Kooy, 2003; 2004; Tan et al, 2009). It is therefore possible that the effects of intravenous or systemic administration of nicotine may involve different intra-NAc neuronal mechanisms, beyond those reported in the present study. Future studies are required to more closely examine these potential differences.

We observed similar patterns of NAshell FSI or MSN activity during conditioning or recall of an acutely rewarding dose of intra-VTA nicotine and following systemic DA receptor blockade. We have reported previously that higher, rewarding doses of intra-VTA nicotine (within the same range used in this report) are independent of DA (Laviolette and van der Kooy, 2003; Tan et al, 2009). Indeed, the acute rewarding properties of intra-VTA nicotine are dependent upon brainstem structures such as the PPT, as lesions of the PPT are capable of blocking the rewarding properties of nicotine (Laviolette et al, 2002). How might nicotineinduced activation of NAshell MSNs produce reward? One possibility may be via indirect projections from the ventral striatum to output structures such as the ventral pallidum (VP). The VP sends a substantial GABAergic projection to the brainstem, including the PPT (Inglis et al, 1994; Rye et al, 1988). Activation of MSN inhibitory outputs to the VP would in turn be expected to inhibit VP inhibitory influences on the PPT. Although future experiments are required to directly test this hypothesis, it is noteworthy that the PPT is critically involved in mediating the rewarding properties of both intra-VTA and systemically administered nicotine (Corrigall et al, 2001; Lança et al, 2000; Laviolette et al, 2002), and is functionally linked to NAshell output pathways (Inglis et al, 1994). Thus, although nicotine reward conditioning induced robust effects on spontaneous FSI vs MSN activity patterns within the NAshell, it is important to note that the VTA contains both DAergic and non-DAergic, largely GABAergic neuronal populations, both of which are activated by nicotine administration (Yin and French, 2000) and send both
DAergic and GABAergic projections to the ventral striatum (Van Bockstaele and Pickel, 1995). One possibility is that higher concentrations of intra-VTA nicotine may recruit inhibitory GABAergic outputs to the NAc, leading to inhibitory effects on striatal interneurons, resulting in indirect disinhibition of MSN output neurons.

In summary, considerable evidence suggests that striatal network activity involves a complex interplay between different populations of MSNs and interneuron populations involved in the processing of either rewarding or aversive motivational information. Our findings provide the first evidence for distinct patterns of NAshell FSI/MSN activity during the acquisition and recall of either rewarding or aversive, nicotine-related behavioral effects and reveal a critical role for DA transmission in the mediation of these bivalent neuronal patterns within the mesolimbic circuit.

\section{FUNDING AND DISCLOSURE}

The authors declare no conflict of interest.

\section{ACKNOWLEDGEMENTS}

This work was supported by Pfizer Canada.

\section{REFERENCES}

Aosaki T, Kiuchi K, Kawaguchi Y (1998). Dopamine D1-like receptor activation excites rat striatal large aspiny neurons in vitro. J Neurosci 18: 5180-5190.

Berger TW, Nisenbaum ES, Stricker EM, Zigmond MJ (1987). Evidence for two functionally distinct subpopulations of neurons within striatum and their differential sensitivity to dopamine. In: Chiodo LAFreeman AS (eds.) Neurophysiology of Dopaminergic Systems. Lakeshore: Detroit, USA, pp 253-284.

Berke JD, Okatan M, Skurski J, Eichenbaum HB (2004). Oscillatory entrainment of striatal neurons in freely moving rats. Neuron 43: 883-896.

Bracci E, Centonze D, Bernardi G, Calabresi P (2002). Dopamine excites fast-spiking interneurons in the striatum. J Neurophysiol 87: 2190-2194.

Cadoni C, Muto T, Di Chiara G (2009). Nicotine differentially affects dopamine transmission in the nucleus accumbens shell and core of Lewis and Fischer 344 rats. Neuropharmacology 57: 496-501.

Caggiula AR, Donny EC, Chaudhri N, Perkins KA, Evans-Martin FF, Sved AF (2002). Importance of nonpharmacological factors in nicotine self-administration. Physiol Behav 77: 683-687.

Chaudhri N, Caggiula AR, Donny EC, Palmatier MI, Liu X, Sved AF (2006). Complex interactions between nicotine and nonpharmacological stimuli reveal multiple roles for nicotine in reinforcement. Psychopharmacology 184: 353-366.

Corrigall WA, Coen KM, Adamson KL (1994). Self-administered nicotine activates the mesolimbic dopamine system through the ventral tegmental area. Brain Res 653: 278-284.

Corrigall WA, Coen KM, Zhang J, Adamson KL (2001). GABA mechanisms in the pedunculopontine tegmental nucleus influence particular aspects of nicotine self-administration selectively in the rat. Psychopharmacology 158: 190-197.

Corrigall WA, Franklin KB, Coen KM, Clarke PB (1992). The mesolimbic dopaminergic system is implicated in the reinforcing effects of nicotine. Psychopharmacology 107: 285-289. 
Creese I, Burt DR, Snyder SH (1976). Dopamine receptor binding predicts clinical and pharmacological potencies of antischizophrenic drugs. Science 192: 481-483.

David V, Besson M, Changeux JP, Granon S, Cazala P (2006). Reinforcing effects of nicotine microinjections into the ventral tegmental area of mice: dependence on cholinergic nicotinic and dopaminergic D1 receptors. Neuropharmacology 50: 1030-1040.

English DF, Ibanez-Sandoval O, Stark E, Tecuapetla F, Buzsaki G, Diesseroth $\mathrm{K}$ et al (2012). GABAergic circuits mediate the reinforcement-related signals of striatal cholinergic interneurons. Nat Neurosci 15: 123-130.

Gage GJ, Stoetzner CR, Wiltschko AB, Berke JD (2010). Selective activation of striatal fast-spiking interneurons during choice execution. Neuron 67: 466-479.

Hu XT, Wachtel SR, Galloway MP, White FJ (1990). Lesions of the nigrostriatal dopamine projection increase the inhibitory effects of D1 and D2 dopamine agonists on caudate-putamen neurons and relieve D2 receptors from the necessity of D1 receptor stimulation. J Neurosci 10: 2318-2329.

Hu XT, White FJ (1997). Dopamine enhances glutamate-induced excitation of rat striatal neurons by cooperative activation of D1 and D2 class receptors. Neurosci Lett 224: 61-65.

Inglis WL, Dunbar JS, Winn P (1994). Outflow from the nucleus accumbens to the pedunculopontine tegmental nucleus: a dissociation between locomotor activity and responding for conditioned reinforcement stimulated by d-amphetamine. Neuroscience 62: 51-64.

Jorenby DE, Steinpreis RE, Sherman JE, Baker TB (1990). Aversion instead of preference learning indicated by nicotine place conditioning in rats. Psychopharmacology 101: 533-538.

Joshua M, Adler A, Mitelman R, Vaadia E, Bergman H (2008). Midbrain dopaminergic neurons and striatal cholinergic interneurons encode the difference between reward and aversive events at different epochs of probalistic classical conditioning trials. J Neurosci 28: 11673-11684.

Kimura M, Rajkowski J, Evarts E (1984). Tonically discharging putamen neurons exhibit set-dependent responses. Proc Natl Acad Sci USA 81: 4998-5001.

Koos T, Tepper JM (1999). Inhibitory control of neostriatal projection neurons by GABAergic interneurons. Nat Neurosci 2: 467-472.

Lansink CS, Goltstein M, Lankelma JV, Pennartz CMA (2010). Fast-spiking interneurons of the rat ventral striatum: temporal coordination of activity with principal cells and responsiveness to reward. Eur J Neurosci 32: 494-508.

Lança AJ, Adamson KL, Coen KM, Chow BL, Corrigall WA (2000). The pedunculopontine tegmental nucleus and the role of cholinergic neurons in nicotine self-administration in the rat: a correlative neuroanatomical and behavioral study. Neuroscience 96: 735-742.

Laviolette SR, Alexson TO, van der Kooy D (2002). Lesions of the tegmental pedunculopontine nucleus block the rewarding effects and reveal the aversive effects of nicotine in the ventral tegmental area. J Neurosci 22: 8653-8660.

Laviolette SR, Lauzon NM, Bishop SF, Sun N, Tan H (2008). Dopamine signaling through D1-like versus D2-like receptors in the nucleus accumbens core versus shell differentially modulates nicotine reward sensitivity. J Neurosci 28: 8025-8033.

Laviolette SR, Nader K, van der Kooy D (2002). Motivational state determines the functional role of the mesolimbic dopamine system in the mediation of opiate reward processes. Behav Brain Res 129: 17-29.

Laviolette SR, van der Kooy D (2004). The neurobiology of nicotine addiction: bridging the gap from molecules to behaviour. Nat Rev Neurosci 5: 55-65.

Laviolette SR, van der Kooy D (2003). Blockade of mesolimbic dopamine transmission dramatically increases sensitivity to the rewarding effects of nicotine in the ventral tegmental area. Mol Psychiatry 8: 50-59.
Ma YY, Henley SM, Toll J, Jentsch D, Evans CJ, Levine MS et al (2013). Drug-primed reinstatement of cocaine seeking in mice: increased excitability of medium-sized spiny neurons in the nucleus accumbens. ASN Neuro 5: 257-271.

Mansvelder HD, Keath JR, McGehee DS (2002). Synaptic mechanisms underlie nicotine-induced excitability of brain reward areas. Neuron 33: 905-919.

Mereu G, Yoon KW, Boi V, Gessa GL, Naes L, Westfall TC (1987). Preferential stimulation of ventral tegmental area dopaminergic neurons by nicotine. Eur J Pharmacol 141: 395-399.

Morra JT, Glick SD, Cheer JF (2010). Neural encoding of psychomotor activation in the nucleus accumbens core, but not the shell, requires cannabinoid receptor signaling. J Neurosci 30: $5102-5107$.

Nisell M, Nomikos GG, Svensson TH (1994). Systemic nicotineinduced dopamine release in the rat nucleus accumbens is regulated by nicotinic receptors in the ventral tegmental area. Synapse 16: 36-44.

Nisenbaum ES, Berger TW (1992). Functionally distinct subpopulations of striatal neurons are differentially regulated by GABAergic and dopaminergic inputs-I. In vivo analysis. Neuroscience 48: 561-578.

Paxinos G, Watson C (2005). The Rat Brain in Stereotaxic Coordinates. 5th edn Academic Press: San Diego, USA.

Perez MF, White FJ, Hu XT (2006). Dopamine D(2) receptor modulation of $\mathrm{K}(+)$ channel activity regulates excitability of nucleus accumbens neurons at different membrane potentials. J Neurophysiol 96: 2217-2228.

Perkins KA (1995). Individual variability in responses to nicotine. Behav Genet 25: 119-132.

Picciotto MR, Corrigall WA (2002). Neuronal systems underlying behaviors related to nicotine addiction: neural circuits and molecular genetics. J Neurosci 22: 3338-3341.

Pisani A, Bonsi P, Centonze D, Calabresi P, Bernardi G (2000). Activation of D2-like dopamine receptors reduces synaptic inputs to striatal cholinergic interneurons. J Neurosci. 20: RC69.

Planert H, Szydlowski SN, Hjorth JJ, Grillner S, Silberberg G (2010). Dynamics of synaptic transmission between fast-spiking interneurons and striatal projection neurons of the direct and indirect pathways. J Neurosci 30: 3499-3507.

Podda MV, Riccardi E, D’Ascenzo M, Azzena GB, Grassi C. (2010). Dopamine D1-like receptor activation depolarizes medium spiny neurons of the mouse nucleus accumbens by inhibiting inwardly rectifying $\mathrm{K}+$ currents through a cAMP-dependent protein kinase A-independent mechanism. Neuroscience 167: 678-690.

Pomerleau OF (1995). Individual differences in sensitivity to nicotine: implications for genetic research on nicotine dependence. Behav Genet 25: 161-177.

Pontieri FE, Tanda G, Orzi F, Di Chiara G (1996). Effects of nicotine on the nucleus accumbens and similarity to those of addictive drugs. Nature 382: 255-257.

Rye DB, Lee HJ, Saper CB, Wainer BH (1988). Medullary and spinal efferents of the penunculopontine tegmental nucleus and adjacent mesopontine tegmentum in the rat. J Comp Neurol 269: 315-341.

Sellings LH, Baharnouri G, McQuade LE, Clarke PB (2008). Rewarding and aversive effects of nicotine are segregated within the nucleus accumbens. Eur J Neurosci 28: 342-352.

Smith AD, Bolam JP (1990). The neural network of the basal ganglia as revealed by the study of synaptic connections of identified neurones. Trends Neurosci 13: 259-265.

Sun N, Chi N, Lauzon N, Bishop S, Tan H, Laviolette SR (2011). Acquisition, extinction, and recall of opiate reward memory are signaled by dynamic neuronal activity patterns in the prefrontal cortex. Cereb Cortex 21: 2665-2680.

Sun N, Laviolette SR (2012). Inactivation of the basolateral amygdala during opiate reward learning disinhibits prelimbic cortical neurons and modulates associative memory extinction. Psychopharmacology 222: 645-661. 
Taha SA, Fields HL (2005). Encoding of palatability and appetitive behaviors by distinct neuronal populations in the nucleus accumbens. J Neurosci 25: 1193-1202.

Tan H, Bishop SF, Lauzon NM, Sun N, Laviolette SR (2009). Chronic nicotine exposure switches the functional role of mesolimbic dopamine transmission in the processing of nicotine's rewarding and aversive effects. Neuropharmacology 56: 741-751.

Tepper JM, Tecuapetla F, Koós T, Ibáñez-Sandoval O (2010). Heterogeneity and diversity of striatal GABAergic interneurons. Front Neuroanat 4: 150.

Threlfell S, Lalic T, Platt NJ, Jennings KA, Deisseroth K, Cragg SJ (2012). Striatal dopamine release is triggered by synchronized activity in cholinergic interneurons. Neuron 75: 58-64.
Van Bockstaele EJ, Pickel VM. (1995). GABA-containing neurons in the ventral tegmental area project to the nucleus accumbens in rat brain. Brain Res 682: 215-221.

Wiltschko AB, Pettibone JR, Berke JD. (2010). Opposite effects of stimulant and antipsychotic drugs on striatal fast-spiking interneurons. Neuropsychopharmacology 35: 1261-1270.

Yin R, French ED (2000). A comparison of the effects of nicotine on dopamine and non-dopamine neurons in the rat ventral tegmental area: an in vitro electrophysiological study. Brain Res Bull 51: 507-514.

Zocchi A, Girlanda E, Varnier G, Sartori I, Zanette L, Wildish GA et al (2003). Dopamine responsiveness to drugs of abuse: a shellcore investigation in the nucleus accumbens of the mouse. Synapse 50: 293-302. 